\title{
Adipose-derived stem cell-derived microvesicles prevent progression to bladder underactivity secondary to long-termed bilateral partial iliac arterial occlusion-induced bladder ischemia
}

\section{Bing-Juin Chiang}

Fu Jen Catholic University College of Medicine https://orcid.org/0000-0003-4398-4997

\section{Su-Han Mao}

National Taiwan Normal University

Shiu-Dong Chung

Far Eastern Memorial Hospital

Chiang-Ting Chien ( $\nabla$ ctchien@ntnu.edu.tw)

National Taiwan Normal University

\section{Research Article}

Keywords: adipose-derived stem cells, ADSC-derived microvesicles, bilateral partial iliac arterial occlusion, underactive bladder, rat

Posted Date: August 26th, 2021

DOl: https://doi.org/10.21203/rs.3.rs-832404/v1

License: (c) (i) This work is licensed under a Creative Commons Attribution 4.0 International License.

Read Full License 


\section{Abstract \\ Background}

The therapeutic effects of adipose-derived stem cells (ADSCs) and ADSC-derived microvesicles (MVs) were investigated in a rat model of bladder ischemia involving long-term (4 weeks) bilateral partial iliac arterial occlusion (BPAO).

\section{Methods}

The study included four groups: sham, BPAO, BPAO + ADSCs, and BPAO + ADSC-derived MVs. ADSCs or ADSC-derived MVs were injected through the femoral artery. Real-time laser speckle contrast imaging evaluated bladder microcirculation following BPAO. A 24-h behavior study and transcystometrogram were conducted after 4 weeks. Bladder histology, immunostaining, and lipid peroxidation assays were performed. The expression of P2X2, P2X3, M2, and M3 receptors, nerve growth factor (NGF), and collagen-1 was evaluated.

\section{Results}

BPAO for 4 weeks significantly reduced bladder microcirculation, prolonged the intercontraction interval, decreased voiding volume, increased residual urine volume, lengthened phase 1 contraction, shortened phase 2 contraction, increased neutrophil infiltration, increased malondialdehyde levels, and altered levels of P2X3 receptors. ADSC-derived MVs significantly ameliorated these effects, increased NGF expression, and decreased collagen-1 expression. ADSCs only improved voiding volume and increased residual urine volume.

\section{Conclusions}

ADSC-derived MVs prevented adverse consequences of long-term BPAO, including detrusor underactivity, bladder ischemia, and oxidative stress. Amelioration of inflammation, altered purinergic signaling, neuronal regeneration, and decreased fibrosis might be involved in the therapeutic mechanism.

\section{Introduction}

Underactive bladder (UAB) is a symptom complex characterized by a slow urinary stream, hesitancy, and straining to void, with or without a feeling of incomplete bladder emptying, sometimes with storage symptoms according to the International Continence Society (ICS) definition [1]. Detrusor underactivity (DU) is a urodynamic definition by ICS that represents low detrusor pressure or short detrusor contraction time, usually in combination with a low urine flow rate, resulting in prolonged bladder emptying and/or a failure to achieve complete bladder emptying within a normal time span [1]. UAB is a clinical syndrome 
that includes DUs. The prevalence of DU ranges from $9 \%$ to $48 \%$ in men and from $12 \%$ to $45 \%$ in older women with non-neurogenic lower urinary tract symptoms [2,3]. Aging is a major cause of UAB and DUs. It causes bladder decrease in blood flow and bladder receptor changes, resulting in clinical and urodynamic changes [4]. Several diseases or conditions, including benign prostate hypertrophy (BPH)related bladder outlet obstruction (BOO), diabetes mellitus (DM), central nervous system (CNS) diseases, arterial atherosclerosis, and infections, also increase in incidence in proportion to age [5]. These diseases or conditions might also contribute to DU/UAB through bladder ischemia, excessive oxidative stress, chronic inflammation, and instability of inhibition signaling. The micturition cycles were then interfered by various mechanisms, including detrusor myogenic failure, bladder afferent nerve dysfunction, bladder efferent nerve dysfunction, and brain/spinal cord dysfunction[6]. Vascular factors have been proven to be one of the most important etiologies of lower urinary tract symptoms (LUTS) [7]. Bladder perfusion was also shown to be associated with the severity of LUTS in transrectal color Doppler ultrasonography and dynamic contrast-enhanced magnetic resonance imaging studies [8, 9]. Severed or prolonged ischemia has been proposed to induce bladder progression from overactive bladder (OAB)/detrusor overactivity (DO) to UAB/DU [4]. Current therapeutic strategies for DU intervention are limited [10]. Existing pharmacologic agents lack sufficient efficacy and fail to achieve improved quality of life [11]. Clean intermittent catheterization remains the gold standard to reduce the adverse consequences of a high PVR and incomplete voiding [10].

Stem cell therapies as regenerative medicine have shown potential in the treatment of diseases that cannot be treated [12]. Several animal studies have shown that ADSCs directly injected into the bladder via laparotomy successfully ameliorated high fructose diet and high cholesterol diet-induced ischemic bladder and associated physiological changes $[13,14]$. Previously, we developed an animal model with bilateral partial iliac arterial occlusion (BPAO) to induce bladder ischemia without confounding metabolic syndrome [15]. The results demonstrated that BPAO is capable of inducing bladder ischemia, oxidative stress, and programmed cell death. In a subsequent study, we found that intra-arterial injection via the femoral artery of adipose tissue-derived stem cells (ADSCs) and ADSC-derived microvesicles (MVs) significantly ameliorated ischemia-related bladder overactivity by attenuating oxidative stress, inflammation, and micturition-related receptors [16]. The results showed a promising therapeutic effect of ADSCs on short-term ischemic bladder dysfunction with bladder overactivity. The study is also the first to demonstrate the therapeutic effect of ADSC-derived MVs on bladder dysfunction. In the current study, we applied the same setting of BPAO with 2 weeks longer duration [16]. We explored the adverse presentation of long-term BPAO. We believe that a longer exposure to bladder ischemia leads to DU. We also evaluated the therapeutic effect of ADSCs and ADSC-derived MVs via intra-arterial injection, which is an easier and minimally invasive way to administer target agents.

\section{Materials And Methods}

Animal Groups and Experimental Design 
Twenty-four adult female Wistar rats weighing 200-240 g were purchased from Bio-LASCO Taiwan (Taipei, Taiwan). They were housed at a constant temperature under a consistent light-dark cycle (light from 07:00 to 18:00) at the Experimental Animal Center of National Taiwan Normal University. All animal surgical and experimental procedures were approved by the Institutional Animal Care and Use Committee of National Taiwan Normal University, College of Life Science (approval No. 106049) and performed in accordance with the guidelines of the National Science Council of the Republic of China (NSC 1997).

The rats were divided into four groups: (1) sham control (sham, $n=6),(2) 4$ weeks of bladder ischemia induced by BPAO (BPAO, $n=6$ ), (3) ADSCs injection through one of the femoral arteries in the week following BPAO and a total of 4 weeks of BPAO-induced bladder ischemia (BPAO + ADSCs, $n=6$ ), and (4) ADSC-derived MVs injection through one of the femoral arteries at the week following BPAO and a total of 4 weeks of BPAO-induced bladder ischemia (BPAO + ADSC-derived MVs, $n=6$ ). Baseline physiological parameters, including urinary frequency, water intake, food intake, urine output (labeled as urine), and feces output (labeled as feces), were recorded and analyzed in R-2100 metabolic cages (Lab Products, MD, USA) for $24 \mathrm{~h}$ in the four groups. Figure $1 \mathrm{a}$ illustrates the induction of the BPAO. The detailed procedures have been described in our previous study [16]. After BPAO, each rat received subcutaneous administration of ketoprofen (Sigma-Aldrich, Darmstadt, GER) $5 \mathrm{mg} / \mathrm{kg}$ once daily for one week. Four weeks after BPAO, the bladder microcirculation was evaluated. After physiological experiments with the metabolic cage, transcystogram, and pelvic nerve activity studies, the bladder was removed. The weight of the bladder was measured. One part of the bladder was cut and reserved for formalin fixation and further staining. Some of the samples were stored at $-70^{\circ} \mathrm{C}$ for protein expression analysis through western blotting and lipid peroxidation (malondialdehyde [MDA]) assay. The details of the respective techniques and studies are addressed in later sections.

Preparation of ADSCs and ADSC-Derived MVs

Adult female Wistar rats weighing 280-300 g were purchased from Bio-LASCO Taiwan (Taipei, Taiwan). The flank adipose tissue was collected under sterile conditions after administering general anesthesia. ADSCs standard isolation was performed as described previously in detail [17]. The adipose tissue was then stirred to aspirate the saline and oil phases. The fat was washed three to five times with $0.1 \mathrm{M}$ phosphate-buffered saline (PBS) (Sigma-Aldrich, Darmstadt, Germany), and the lower phase was discarded until clear. Collagenase was added after the final upper phase was collected. The solution was incubated for $1-4 \mathrm{~h}$ at $37^{\circ} \mathrm{C}$ on a shaker. Next,10\% fetal bovine serum (FBS) (Invitrogen, MA, USA) was added to the tube to neutralize collagenase. The fluid in the tube containing the digested fat was centrifuged at $800 \times \mathrm{g}$ for $10 \mathrm{~min}$. The supernatant containing liquid, lipid, and floating adipocytes was aspirated to obtain the left stromal vascular fraction (SVF) pellet. We used $160 \mathrm{mM} \mathrm{NH}_{4} \mathrm{Cl}$ to suspend the SVF pellet, which was incubated for 10 min at room temperature (RT). The pellet was centrifuged at $400 \times$ $\mathrm{g}$ for 10 min at RT after incubation. The final pelleted fraction of mononuclear cells was resuspended in Dulbecco's modified Eagle's medium (DMEM) (Sigma-Aldrich, Darmstadt, Germany) supplemented with $40 \%$ FBS, penicillin-streptomycin, and $10 \mathrm{ng} / \mathrm{mL}$ of epidermal growth factor (Invitrogen, MA, USA) and incubated on a petri dish overnight to select adherent cells. The remaining debris and cells were removed 
the next day, and the plate was washed with PBS. The ADSCs were then maintained in low-glucose DMEM supplemented with $10 \% \mathrm{FBS}, 1 \%$ penicillin-streptomycin, and L-glutamine at $5 \% \mathrm{CO}_{2}$ and $37^{\circ} \mathrm{C}$. ADSCs were maintained in a T75 flask and passaged until 80-90\% confluence. ADSCs were trypsinized and counted to obtain $5 \times 10^{5}$ cells for treatment.

Preparation of ADSC-derived MVs

ADSC-derived MVs were isolated as previously described [17, 18]. Briefly, MVs were purified by differential ultracentrifugation. ADSCs ( $5 \times 10^{5}$ cells) were cultured in low-glucose DMEM without FBS and supplemented with $0.5 \%$ BSA overnight. The clear conditioned medium was transferred to centrifuge tubes and centrifuged for $10 \mathrm{~min}$ at $300 \times \mathrm{g}$ and $4^{\circ} \mathrm{C}$. The pellets filled with dead cells and cell debris were removed; the supernatant was retained. The supernatant was then centrifuged three times for $10 \mathrm{~min}$ at $2000 \times \mathrm{g}, 30 \mathrm{~min}$ at $10,000 \times \mathrm{g}$, and $30 \mathrm{~min}$ at $10,000 \times \mathrm{g}$ at $4^{\circ} \mathrm{C}$. The final supernatant was discarded, and isolated MVs were suspended in Dulbecco's phosphate-buffered saline (DPBS) (Sigma-Aldrich, Darmstadt, Germany) and then re-centrifuged for $30 \mathrm{~min}$ at $10,000 \times \mathrm{g}$ at $4^{\circ} \mathrm{C}$ to remove contaminating proteins. We collected the pellet containing MVs and resuspended them in DPBS. MVs were washed and ultra-centrifuged at $10,000 \times \mathrm{g}$ for $60 \mathrm{~min}$ at $4^{\circ} \mathrm{C}$. The supernatant was discarded; the MVs pellets were resuspended in DPBS for later use. MVs purity was assayed with CD29 (Becton Dickinson, NJ, USA) and morphology was analyzed using a scanning electron microscope (JEOL-6500F, JEOL, Japan). The purified vesicles under this protocol yielded high CD29-positive expression, spheroid morphology, and 70 $\mathrm{nm}$ size exosomes by scanning electron microscopy [16]. ADSC-derived MVs were injected through the femoral artery at a dose of $100 \mu \mathrm{g} \mathrm{MV}$ proteins, and approximately $5 \times 10^{5} \mathrm{ADSC}$ were released overnight.

Measurement of Bladder Microcirculation

A full-field laser perfusion imager (Moor FLPI; Moor Instruments, Devon, UK) was used to quantify the intensity of microcirculatory blood flow in the bladder in the four groups 4 weeks after the experiments. The details were described previously [16]. This imager uses real-time laser speckle contrast continuous non-contact imaging, which exploits the random speckle pattern generated when the tissue is illuminated by laser light. The microcirculatory blood flow intensity in the region of interest was recorded as "Flux" with perfusion units. This is related to the concentration and average speed of moving red blood cells in the region of interest. When there is a high level of movement (fast flow), the changing pattern becomes more blurred, and the contrast in that region is reduced accordingly. Therefore, low contrast is related to high flow, and high contrast is related to low flow. The contrast image produces 16-color images that are correlated with blood flow in the field of interest. A low level of blood flow was recorded in blue, whereas a high level of blood flow was recorded in red color (range, $0-1000$ ). Images were recorded and analyzed in real time using Moor FLPI 3.0 software (Moor Instruments, Devon, UK).

Evaluation of Transcystometrogram and pelvic nerve activity 
All animals were anesthetized with subcutaneous urethane, which is known to allow full-reflex bladder contractions [19]. We used a transcystometrogram to evaluate variations in micturition parameters. Briefly, rats were anesthetized by subcutaneous injection of urethane $(1.2 \mathrm{~g} / \mathrm{kg}$ body weight). The bladder was exposed through a lower abdominal midline incision. A PE-50 catheter was then inserted through the apex of the bladder dome and connected through a T-tube to a P23 ID infusion pump and pressure transducer (Gould-Statham, MA, USA). During the experiment, the infusion rate was maintained at 1.2 $\mathrm{mL} / \mathrm{h}$, and intravesical pressure (IVP) was continuously recorded using an ADI system (Power-Lab/16S, ADI Instruments, Pty., Ltd., Castle Hill, Australia). The bladder was emptied. Three reproducible micturition cycles were recorded following bladder emptying. The following parameters of bladder activity were recorded: intercontraction interval $(\mathrm{ICl})$, the time between two micturition cycles identified based on active contractions (> $>15 \mathrm{mmHg}$ ), average urine amount per micturition (labeled as average urine amount), bladder volume, amplitude of IVP on micturition contraction (labeled as amplitude), and residual urine amount (labeled as residual urine). Additionally, we recorded and analyzed contraction phases during a micturition cycle: an initial rise in IVP (phase 1) and a series of high-frequency oscillations (HFOs; phase 2). Non-voiding contractions (NVCs) were characterized by an increase in pressure of $>20 \%$ from baseline, defined as the bladder pressure immediately before voiding. This was observed as an increase in pressure, which did not result in voids [20]. The number, mean amplitude, and maximum amplitude were analyzed.

The electrophysiological techniques used to record pelvic afferent nerve activity (PANA) and pelvic efferent nerve activity (PENA) have been described previously [19, 21]. Briefly, these activities were recorded simultaneously from two vesical branches of the pelvic nerve branch, which were microdissected from the surface of the bladder. The firing activities of each neural filament were recorded by placing the nerve fibers in parallel with two pairs of thin bipolar stainless steel electrodes. The electrodes and bladder nerves were bathed in a pool of warm $\left(37^{\circ} \mathrm{C}\right)$ paraffin oil to prevent nerve drying. PANA was recorded after crushing the nerve central to the recording site to eliminate efferent firing. PENA was recorded when the bladder nerve was crushed distal to the recording site. The electrical signals were amplified 20,000-fold and filtered (high-frequency cut-off at $3000 \mathrm{~Hz}$, low-frequency cut-off at $30 \mathrm{~Hz}$ ) using a Grass model P511 a.c. preamplifier (Valley View, OH U.S.A.) and continuously recorded and displayed on an ADI system (Power-Lab/16S, ADI Instruments, Pty., Ltd., Castle Hill, Australia). The amplified signals (spikes) were transformed by a window discriminator 121 (World Precision Instruments, Sarasota, FL), which was set to count the total number of spikes per second. The background activity, which could be caused by nerve contact with electrodes, nerve damage during handling, and the equipment itself, was excluded from the window discriminator by adjustment of the threshold voltage. PE-50 catheters were placed in the left femoral artery to measure arterial blood pressure (ABP). ABP was recorded in an ADI system (Power-Lab/16S; ADI Instruments, Pty., Ltd., Castle Hill, Australia) with a transducer (Gould-Statham; Quincy, MA, USA). Three reproducible micturition cycles on the transcystogram were simultaneously recorded following bladder emptying. The following parameters were recorded: ABP (mmHg), IVP (mmHg), PENA( $\mu \mathrm{v}), \operatorname{PANA}(\mu \mathrm{v})$, cumulated PENA (cPENA) (spikes/s), and 
cumulated PANA (cPANA) (spikes/s). The voltage changes (\%) of PENA ( $\mu \mathrm{v})$ and PANA ( $\mu \mathrm{v}), \mathrm{cPENA}$ (spikes/s), and cPANA(spikes/s) between the micturition phase and baseline were analyzed.

Morphological Staining

A portion of the bladder was cut and fixed in a 10\% neutral-buffered formalin solution. The tissues were dehydrated in graded ethanol and embedded in paraffin. Sections $(4 \mu \mathrm{m})$ of the bladder were stained with hematoxylin and eosin to evaluate the extent of neutrophils. To prepare slides for immunohistochemical examination, slides were blocked with $5 \%$ bovine serum (Cat\# ALB001; BioShop, Ontario, Canada) in PBS with $0.3 \%$ Triton-X 100 (Sigma-Aldrich, MO, USA) for $1 \mathrm{~h}$. The slides were incubated overnight with primary antibodies prepared using $1 \%$ normal donkey serum and $0.1 \%$ Triton-X 100 in 0.1 M PBS at RT. Collagen I was labeled using a rabbit monoclonal antibody (bs-10423R; Bioss, MA, USA) purified at a working dilution of 1:250. The secondary antibody goat anti-rabbit IgG H\&L (NEF812001EA; Invitrogen, MA, USA) was then applied at a dilution of 1:500. The samples were counter-stained with haematoxylin. Nerve growth factor (NGF) was labeled using a rabbit monoclonal antibody (ab52918; Abcam, Cambridge, UK) purified at a working dilution of 1:250. The secondary antibody goat anti-rabbit IgG H\&L (NEF812001EA; Invitrogen, MA, USA) was then applied at a dilution of 1:500. The samples were counterstained with haematoxylin. Antigen retrieval was performed using Tris-EDTA buffer (pH 9.0). PBS was used instead of primary antibody as a negative control.

Western Blot and Biochemical Evaluation

Expression levels of purinergic P2X2 and P2X3 receptors, muscarinic M2 and M3 receptors, NGF, and collagen-I were analyzed by western blotting as described previously [22-24]. Bladder samples were homogenized using a prechilled mortar and pestle in an extraction buffer containing $10 \mathrm{mM} \mathrm{Tris-HCl}(\mathrm{pH}$ 7.6), $140 \mathrm{mM} \mathrm{NaCl}, 1 \mathrm{mM}$ phenylmethylsulfonyl fluoride, $1 \% \mathrm{NP}-40,0.5 \%$ deoxycholate, $2 \% \beta$ mercaptoethanol, $10 \mathrm{mg} / \mathrm{mL}$ pepstatin $\mathrm{A}$, and $10 \mathrm{mg} / \mathrm{mL}$ aprotinin. The mixture was completely homogenized by vortexing and maintained at $4^{\circ} \mathrm{C}$ for $30 \mathrm{~min}$. The homogenate was centrifuged at $12,000 \times \mathrm{g}$ for $20 \mathrm{~min}$ at $4^{\circ} \mathrm{C}$. The supernatant was collected; the protein concentration was determined using a Bio-Rad protein assay kit (BioRad Laboratories, CA, USA). Antibodies raised against P2X2 receptors (ab10266; Abcam, Cambridge, UK), P2X3 receptors (RA141399; Neuromics, MN, USA), M2 receptors (nb120-2805; Novus Biologi-cals, CO, USA), M3 receptors (ab87199; Abcam, Cambridge, UK), NGF (ab6199; Abcam, Cambridge, UK), and collagen-1 (bs-10423R; Bioss, MA, USA) were used. Sodium dodecyl sulfate-polyacrylamide gel electrophoresis was performed using $10 \%$ separation gels in the absence of urea and Coomassie brilliant blue staining. For immunoblotting, proteins were transferred to Immobilon polyvinylidene difluoride membranes (Millipore, MA, USA) for $18 \mathrm{~h}$ at $100 \mathrm{~mA}$ in a Miniprotean III transfer tank (BioRad, CA, USA). Immunoreactive bands were detected by incubation with the aforementioned antibodies, followed by incubation with an alkaline phosphatase-labeled secondary antibody and western Lightning Plus-ECL stock solution (PerkinElmer, MA, USA) for 1 min at RT. The density of the band was semi-quantitatively determined by densitometry using an image analysis system 
(ImageJ; National Institutes of Health, USA). MDA concentration was determined using a lipid peroxidation assay kit (ab118970; BioVision, CA, USA).

\section{Statistical Analysis}

GraphPad Prism 6 (GraphPad Software, CA, USA) was used. All values are expressed as the mean \pm standard error of the mean. Differences between groups were examined using one-way analysis of variance, followed by Duncan's multiple-range test. Differences within the groups were examined using paired t-tests. Differences were considered significant at $p<0.05$.

\section{Results}

ADSC-derived MVs Mitigated BPAO-induced Bladder Ischemia

Figure $1 \mathrm{~b}$ shows the real-time measurements of the bladder microcirculation. A significant redistribution in the color of the bladder was associated with a decline in high-flow areas (red) and an increase in lowflow areas in the BPAO group compared with the sham group $(p<0.05$, Fig. $1 c)$. The BPAO + ADSC-derived $M V$ group exhibited significant mitigation of reduced microcirculation following $B P A O(p<0.05$, Fig. $1 c)$.

ADSCs and ADSC-derived MVs Ameliorated BPAO-induced Bladder Underactivity

Figure 2 illustrates the physiological parameters determined in the metabolic cage study. Urinary frequency was significantly lower in the BPAO group than in the sham group $(p<0.05$, Fig. $2 b)$. ADSCderived MVs significantly increased urinary frequency compared to the BPAO group ( $p<0.05$, Fig. $2 b)$. The amount of water intake, food intake, urine output, and feces output did not significantly differ among the four groups (Fig. 2c-f).

Figure 3a shows the IVP patterns on transcystometrogram. A higher ICI was observed in the BPAO group than in the sham group ( $p<0.05$; Fig. $3 b)$. The ICI in the BPAO + ADSC-derived MVs group was lower than that in the BPAO group ( $p<0.05$; Fig. 3b). The amplitude was not significantly different between the groups (Fig. 3c). The average urine amount in the BPAO group was significantly lower than that in the sham group ( $p<0.05$, Fig. $3 d)$. The average urine amount in the BPAO + ADSCs and BPAO + ADSC-derived MVs groups was significantly higher than that in the BPAO group $(p<0.05$, Fig. $3 d)$. The residual urine amount was significantly higher in the BPAO group than in the sham group $(p<0.05$; Fig. 3e). The residual urine amount was significantly lower in the BPAO + ADSCs and BPAO + ADSC-derived MVs groups than in the BPAO group ( $p<0.05$; Fig. 3e). Bladder volume was not significantly different (Fig. $3 f$ ). The bladder weights in the BPAO and BPAO + ADSCs groups were significantly higher than those in the sham group. $(p<0.05$; Fig. $3 g)$.

Figure 4a shows the micturition contraction patterns on the transcystometrogram. The duration of phase 1 contraction in the BPAO group was significantly higher than that in the sham group $(p<0.05$, Fig. $4 b)$. The duration of phase 1 contraction in the BPAO + ADSC-derived MVs group was significantly lower than 
that in the BPAO group ( $p<0.05$, Fig. $4 b)$. The duration of phase $2 \mathrm{HFO}$ in the BPAO group was significantly lower than that in the sham group $(p<0.05$, Fig. $4 c)$. The duration of phase 2 HFOs in the BPAO + ADSC-derived MVs group was significantly higher than that in the BPAO group $(p<0.05$, Fig. $4 c)$.

Figure 5a shows the NVCs observed through the transcystometrogram. The number of NVCs did not significantly differ between groups (Fig. $5 b$ ). The mean amplitude of the NVCs did not significantly differ between the groups (Fig. 5c). The maximum amplitude of NVCs was significantly higher in the BPAO group than in the sham group $(p<0.05$; Fig. $5 d$ ). The maximum amplitude of NVCs was significantly lower in the BPAO + ADSC-derived MVs group than in the BPAO group $(p<0.05$; Fig. $5 d)$.

ADSC-Derived MVs Ameliorated BPAO-Induced diminished PANA/PENA and Increased level of NGF expression

Figure 6 demonstrates the PENA and PANA in the separated group. Figure 6a shows the integrated graph recording of ABP (mmHg), IVP (mmHg), PENA( $\mu \mathrm{v}), \operatorname{PANA}(\mu \mathrm{v})$, cPENA (spikes/s), and cPANA (spikes/s). The changes in the micturition phase and baseline tended to be lower in the BPAO group $(p>0.05$, Fig. $6 \mathrm{~b})$. The changes in the PENA ( $\mu \mathrm{v})$ micturition phase and baseline were significantly lower in the BPAO group than in the sham group $(p<0.05$, Fig. $6 c)$. The changes in the PENA $(\mu v)$ were significantly higher in the BPAO + ADSC-derived MVs group than in the BPAO group $(p<0.05$, Fig. $6 c)$. The cPANA per micturition in the BPAO group was significantly lower than that in the sham group $(p<0.05$, Fig. $6 d)$. The cPANA per micturition in the BPAO + ADSCs group and BPAO + ADSC-derived MVs was significantly higher than that in the sham group ( $p<0.05$, Fig. $6 d$ ). The CPENA per micturition in the BPAO group was significantly lower than that in the sham group ( $p<0.05$, Fig. $6 e)$. The cPENA per micturition in BPAO + ADSC-derived MVs was significantly higher than that in the sham group $(p<0.05$, Fig. $6 e)$.

Figure 7a shows the results of NGF staining. Image analysis results showed that NGF density was higher in the BPAO + ADSC-derived MVs group than in the BPAO group ( $p<0.05$; Fig. 7a). NGF density in the BPAO + ADSCs group tended to be higher than that in the BPAO group (Fig. 7a). Western blot results showed significantly increased NGF expression in the BPAO + ADSCs group and BPAO + ADSC-derived MVs group compared with the sham group ( $p<0.05$; Fig. $7 b$ ). The level of NGF expression in the BPAO + ADSC-derived MVs group was significantly higher than that in the BPAO group $(p<0.05$; Fig. $7 b)$.

ADSC-derived MVs influenced the purinergic signaling caused by BPAO

The expression of the P2X2 receptor did not significantly differ between the groups (Fig. 8a). Compared with the sham group, the BPAO group exhibited significantly decreased P2X3 receptors $(p<0.05 ;$ Fig. $8 b)$. The expression of the $\mathrm{P} 2 \mathrm{X} 3$ receptor was significantly higher in the BPAO + ADSC-derived MVs group than in the BAPO group ( $p<0.05$; Fig. $8 b$ ). The expression of M2 and M3 receptors did not differ significantly between the groups (Fig. 8c, d).

ADSCs and ADSC-derived MVs ameliorated inflammatory cell infiltration and oxidative stress 
Figure 9a shows the results of H\&E staining for the four groups, and Fig. 9b presents an analysis of neutrophil counts. The results showed that the number of infiltrated neutrophils in the BPAO and BPAO + ADSCs groups was higher than that in the sham group ( $p<0.05$; Fig. 9b). The neutrophil count was significantly lower in the BPAO + ADSCs and BPAO + ADSC-derived MVs groups than in the BPAO group $(p<0.05$; Fig. $9 b)$. The results of the MDA assay showed that the BPAO group had a significantly higher level of MDA than the other groups $(p<0.05$; Fig. 10).

ADSC-derived MVs decreased the collagen-1 deposition

Figure 11 shows collagen-1 staining and expression in the separated groups. Figure 11a shows that the collagen-1 density in the BPAO + ADSC-derived MVs group was slightly lower than that in the other groups ( $p>0.05$, Fig. 11a). Western blotting showed that the level of collagen-1 in the BPAO + ADSC-derived MVs group was significantly lower than that in the BPAO group $(p<0.05$; Fig. 11b). The level of Collagen-1 $(210 \mathrm{kDa})$ expression in and BPAO + ADSC-derived MVs group had a trend of lower than BPAO group ( $p>$ 0.05 , Fig. 11b).

\section{Discussions}

The link between OAB and UAB was reviewed in 2014 [25]. It was postulated that patients with chronic untreated or treatment-refractory $O A B$ have a significant risk of progression to $U A B$. $O A B$ and $U A B$ also share common etiologies, including BOO, DM, and neurological diseases. These conditions may contribute to repeated bladder ischemia and reperfusion (I/R) injury [26]. Severe and prolonged ischemia with excessive oxidative stress could possibly result in $O A B$, followed by detrusor hyperactivity/impaired contractility (DHIC) and UAB. According to our results, long-term BPAO (4 weeks) induced prolonged bladder ischemia with extension of $\mathrm{ICl}$, decreased voiding amount, increased residual urine amount that resembles human DU. Our previous study demonstrated that short-term BPAO for 2 weeks induced bladder overactivity [16]. These results prove the chronological relevance between OAB and UAB. Compared with other ischemic bladder models, blood flow of the bilateral common iliac arteries can be quantified and simply adjusted by changing the size of needles in the BPAO model [16]. The BPAO model appears to be a more stable model for mimicking sequential changes from DO to DU.

The results showed significant alteration of phasic contraction following BPAO for 4 weeks compared to a previous study with BPAO for 2 weeks. Prolonged bladder ischemia induces lengthening phase 1 contraction and shortening phase 2 contractions. Within phasic 1 contraction, IVP rises as a consequence of tonic PENA containing purinergic and cholinergic components [27]. The increased level of P2X3 receptor in the BPAO group might play a role in the alteration of micturition reflex. Phasic 2 contraction with the bursting PENA is responsible for HFOs in IVP and bladder emptying [27]. The significantly depressed PENA during phasic 2 contraction in the BPAO group is compatible with the shortening phase 2 contraction and lower voiding efficiency in the BPAO group. These results suggest that the alteration of neural electro-signaling resulting from potential nerve injury is responsible for bladder ischemia-related DU. The number and mean amplitude of the NVCs did not significantly differ between the groups. Only 
the maximum amplitude of the NVCs increased after BPAO for four weeks. Additionally, the results of urinary frequency and $\mathrm{ICI}$ reversed compared to those of a previous study [16]. BPAO for 4 weeks still contributed to inflammatory cell infiltration and oxidative stress with increased MDA levels. This phenomenon evidenced BPAO with prolonged bladder ischemia-induced bladder from DO to DU.

Compared with previous studies, ADSC-derived MVs still affected the recovery of bladder microcirculation even 4 weeks after BPAO [16]. ADSC-derived MVs seem to be more effective in treating bladder ischemia than ADSCs. ADSC-derived MVs recovered the urinary frequency, ICl, average urine amount, residual urine amount, patterns of phasic contractions, changes in PENA, and spike numbers of PANA/PENA during micturition. In summary, ADSC-derived MVs can prevent adverse physiological changes related to bladder ischemia. The underlying therapeutic mechanisms include recovery of microcirculation, amelioration of inflammatory cell infiltration, alleviation of oxidative stress, decreased collagen-1 deposition, and possible neuronal regeneration with increased levels of NGF. NGF is a well-known neurotrophic factor that supports the survival, differentiation, and maintenance of peripheral nerve regeneration [28]. The proposed neuronal protective mechanism of NGF induced by ADSC-derived MVs requires further research. In contrast, ADSCs had no effect on microcirculation, patterns of phasic contraction, NVCs, and level of purinergic receptors, although the average urine amount and residual urine amount were improved following ADSCs administration. Partially alleviating inflammation response, recovery of spike number on PANA, and neuronal regeneration induced by increased NGF levels might be involved in the therapeutic mechanisms. Bladder injection with ADSCs and de-ligation of artificial BOO were shown to be effective in treating BOO-induced DU model by recovering pathophysiological remodeling of smooth muscles and connective tissues [29]. Bladder injection with adipocyte-derived dedifferentiated fat cells can differentiate into smooth muscle cell lineages and contribute to bladder tissue regeneration in a cryoinjury mouse model [30]. Tail vein injection with ADSCs ameliorates diabetic bladder dysfunction in a type II diabetic rat model by reducing apoptosis and preserving the suburothelial capillary network [13]. Mesenchymal stem cells (MSCs) are believed to have regeneration ability by expressing growth factors for healing effects, limiting inflammation by releasing cytokines, altering immune responses by secreting immunomodulatory proteins, enhancing responses from endogenous repair cells, and directly serving as mature functional cells [31]. In our both studies reproduced part of the potential therapeutic effects of ADSCs on BPAO-induced DO and DU models. Physiological changes with underlying mechanisms following BPAO might differ from other animal DU models [32]. The effects of different types of stem cells may also vary among different settings. According to the current settings, the ADSCs and ADSCderived MVs were administered the week following BPAO. The regeneration ability of ADSCs declined with the duration of ischemic conditions, especially the DU status. Thus, identifying the critical timing of intervention with stem cell therapies and the cessation of disease status is imperative.

Stem cell therapies have been extensively evaluated in clinical and basic studies. Stress urinary incontinence treated with various types of cell therapies has been the most investigated in the urology field. To date, muscle-derived stem cells and ADSCs have the advantages of abundance and ease of availability [33]. ADSCs can be acquired and injected in a single procedure using a commercial kit with reliable quality in the current era [34]. To ease the risks of tumorigenesis, MSC-derived extracellular 
vesicles (EVs), which include exosomes and MVs, have been studied for their role in various fields. Several studies have demonstrated that MSC-derived exosomes and MVs recapitulate to a large extent the immensely broad therapeutic effects previously attributed to MSCs [31]. These MVs and exosomes are capable of transferring cytokines, growth factors, microRNAs, and proteins. MVs $(0.1-1 \mu \mathrm{m})$ and exosomes (50-150 nm) are small and circulate readily whereas MSCs are too large to circulate easily through capillaries [18]. MSCs could be arrested at microvessel bifurcations due to size obstruction [35]. This might be the reason that the therapeutic effect of ADSC-derived MVs is better than that of ADSCs with an intra-arterial injection route in the current study.

The disadvantages of MVs and exosomes are their static properties and cannot be produced in vivo, as may be possible when transplanting stem cells [31]. Not all MVs and exosomes derived from different individuals are created equally, but depend on the condition of the original stem cells. Thus we used exogenous ADSCs and ADSC-derived MVs prepared from the same ADSCs and their supernatants to evaluate the therapeutic effect equally. Exogenous MSCs from Wistar rats are immunologically tolerable and can be used as donor cells for exogenous cell therapy [36]. From a clinical perspective, studies using autologous ADSCs transplantation and their derived MVs are warranted. Various settings including timing, route, dosage of stem cells, and their derived MVs and exosomes are the future work for exerting their most efficacious and safe. Bladder transplantation is not feasible in the current era. Once the bladder enters the stage of underactivity, there is no effective therapeutic agents. Thus exploration of effective and feasible regenerative medicine or stem cell related therapies is imminent.

\section{Conclusions}

BPAO with prolonged bladder ischemia induced includes DU with extension of $\mathrm{ICl}$, decreased voiding amount, and increased residual urine amount. Inflammation, enhanced oxidative stress, decreased pelvic nerve activity, and altered phasic contractions may be involved in the underlying etiology. Treatment with ADSC-derived MVs exerted better efficacy through femoral artery injection than ADSCs in preventing the adverse effects progressing to DU. To our knowledge, this is the first study to show the potential of ADSCderived MVs in the treatment of ischemia-related DU/UAB. The results suggested ADSCs and ADSCderived MVs may hold promise in the treatment of DU/UAB induced by ischemia.

\section{Abbreviations}

ABP: arterial blood pressure

ADSC: adipose-derived stem cells

BPAO: bilateral partial iliac arterial occlusion

$\mathrm{BPH}$ : benign prostate hypertrophy

BOO: bladder outlet obstruction 
cPANA :cumulated PANA

CPENA: cumulated PENA

DHIC: detrusor hyperactivity/impaired contractility

DO: detrusor overactivity

DU: detrusor underactivity

EVs: extracellular vesicles

HFOs: high-frequency oscillations

ICl: intercontraction interval

I/R: ischemia and reperfusion

IVP: intravesical pressure

LUTS: lower urinary tract symptoms

MDA: malondialdehyde

MSC: mesenchymal stem cell

MVs: microvesicles

NGF: nerve growth factor

NVCs: non-voiding contractions

OAB: overactive bladder

PANA: pelvic afferent nerve activity

PENA: pelvic efferent nerve activity

UAB: underactive bladder

\section{Declarations}

\section{Availability of data and materials}

The datasets used and/or analyzed during the current study are available from the corresponding author on reasonable request. 
Acknowledgements

We would like to thank Editage (www.editage.com) for English language editing and funding support by Taiwan Ministry of Technology and Science and Cardinal Tien Hospital.

\section{Funding}

This study received the following grants: CTH108A-2A20, CTH109A-2204, CTH110A-FJU-29, 106-2314-B567-002-, 107-2314-B-567-002-MY2, 109-2314-B-567 -002 -, and 110-2314-B-567 -002 -MY3

\section{Author information}

\section{Affiliations}

College of Medicine, Fu-Jen Catholic University, No.510, Zhongzheng Rd., Xinzhuang Dist., New Taipei City 24205, Taiwan, R.O.C.

Bing-Juin Chiang and Su-Han Mao

Department of Urology, Cardinal Tien Hospital, No.362, Zhongzheng Rd., Xindian Dist., New Taipei City 23148, Taiwan R.O.C.

Bing-Juin Chiang and Su-Han Mao

Department of Life Science, College of Science, National Taiwan Normal University, No.88 Ting-Chow Rd, Sec 4, Taipei, Taiwan, 116, R.O.C.

Bing-Juin Chiang, Su-Han Mao, and Chiang-Ting Chien

Division of Urology, Department of Surgery, Far-Eastern Memorial Hospital, No.21, Sec. 2, Nanya S. Rd., Banciao Dist., New Taipei City 220, Taiwan, R.O.C.

Shiu-Dong Chung

Graduate Program in Biomedical Informatics, College of Informatics, Yuan-Ze University, No.135 YuanTung Road, Chung-Li, Taiwan 32003, R.O.C.

Shiu-Dong Chung

\section{Contributions}

BJC and CTC conceived and designed the study. SHM performed the experiments. SHM analyzed and interpreted the data. BJC drafted the article. BJC, SHM, SDC, and CTC revised the article critically for important intellectual content. The authors read and approved the final manuscript.

\section{Corresponding authors}


Correspondence to Shiu-Dong Chung and Chiang-Ting Chien

\section{Ethics approval and consent to participate}

All animal surgical and experimental procedures were approved by the Institutional Animal Care and Use Committee of National Taiwan Normal University, College of Life Science (approval No. 106049) and performed in accordance with the guidelines of the National Science Council of the Republic of China.

\section{Consent for publication}

Not applicable.

\section{Competing interests}

The authors declare that they have no competing interests.

\section{References}

1. Chapple $C R$, Osman NI, Birder L, Dmochowski R, Drake MJ. Terminology report from the International Continence Society (ICS) Working Group on Underactive Bladder (UAB). 2018, 37(8):2928-2931.

2. Jeong SJ, Kim HJ, Lee YJ, Lee JK, Lee BK, Choo YM, Oh JJ, Lee SC, Jeong CW, Yoon CY, et al. Prevalence and Clinical Features of Detrusor Underactivity among Elderly with Lower Urinary Tract Symptoms: A Comparison between Men and Women. Korean journal of urology. 2012;53(5):342-8.

3. Miyazato M, Yoshimura N, Chancellor MB. The other bladder syndrome: underactive bladder. Rev Urol. 2013;15(1):11-22.

4. Andersson KE, Boedtkjer DB, Forman A. The link between vascular dysfunction, bladder ischemia, and aging bladder dysfunction. Ther Adv Urol. 2017;9(1):11-27.

5. Taylor JA 3rd, Kuchel GA. Detrusor underactivity: Clinical features and pathogenesis of an underdiagnosed geriatric condition. J Am Geriatr Soc. 2006;54(12):1920-32.

6. Aizawa N, Igawa Y. Pathophysiology of the underactive bladder. Investigative clinical urology. 2017;58(Suppl 2):82-s89.

7. Ponholzer A, Temml C, Wehrberger C, Marszalek M, Madersbacher S. The association between vascular risk factors and lower urinary tract symptoms in both sexes. European urology. 2006;50(3):581-6.

8. Pinggera GM, Mitterberger M, Steiner E, Pallwein L, Frauscher F, Aigner F, Bartsch G, Strasser H. Association of lower urinary tract symptoms and chronic ischaemia of the lower urinary tract in elderly women and men: assessment using colour Doppler ultrasonography. BJU Int. 2008;102(4):470-4.

9. De EJ, Hou P, Estrera AL, Sdringola S, Kramer LA, Graves DE, Westney OL. Pelvic ischemia is measurable and symptomatic in patients with coronary artery disease: a novel application of dynamic contrast-enhanced magnetic resonance imaging. J Sex Med. 2008;5(11):2635-45. 
10. Guidelines EAU. vol. Edn: presented at the EAU Annual Congress Milan 2021.

11. Drake MJ, Williams J, Bijos DA. Voiding dysfunction due to detrusor underactivity: an overview. Nature reviews Urology. 2014;11(8):454-64.

12. Rajabzadeh N, Fathi E, Farahzadi R. Stem cell-based regenerative medicine. Stem cell investigation. 2019;6:19.

13. Zhang H, Qiu X, Shindel AW, Ning H, Ferretti L, Jin X, Lin G, Lin CS, Lue TF. Adipose tissue-derived stem cells ameliorate diabetic bladder dysfunction in a type II diabetic rat model. Stem Cells Dev. 2012;21(9):1391-400.

14. Huang YC, Shindel AW, Ning H, Lin G, Harraz AM, Wang G, Garcia M, Lue TF, Lin CS. Adipose derived stem cells ameliorate hyperlipidemia associated detrusor overactivity in a rat model. The Journal of urology. 2010;183(3):1232-40.

15. Tai HC, Chung SD, Chien CT, Yu HJ. Sulforaphane Improves Ischemia-Induced Detrusor Overactivity by Downregulating the Enhancement of Associated Endoplasmic Reticulum Stress, Autophagy, and Apoptosis in Rat Bladder. Scientific reports. 2016;6:36110.

16. Chiang B-J, Liao C-H, Mao S-H, Chien C-T. Adipose-Derived Stem Cells and Their Derived Microvesicles Ameliorate Detrusor Overactivity Secondary to Bilateral Partial Iliac Arterial OcclusionInduced Bladder Ischemia. Int J Mol Sci. 2021;22(13):7000.

17. Francis MP, Sachs PC, Elmore LW, Holt SE. Isolating adipose-derived mesenchymal stem cells from lipoaspirate blood and saline fraction. Organogenesis. 2010;6(1):11-4.

18. Sluijter JPG, Davidson SM, Boulanger CM, Buzás El, de Kleijn DPV, Engel FB, Giricz Z, Hausenloy DJ, Kishore R, Lecour S, et al. Extracellular vesicles in diagnostics and therapy of the ischaemic heart: Position Paper from the Working Group on Cellular Biology of the Heart of the European Society of Cardiology. Cardiovascular research. 2018;114(1):19-34.

19. Chien CT, Yu HJ, Lin TB, Lai MK, Hsu SM. Substance P via NK1 receptor facilitates hyperactive bladder afferent signaling via action of ROS. American journal of physiology Renal physiology. 2003;284(4):F840-51.

20. Balog BM, Tangada A, Sheth P, Song QX, Couri BM, Porras LL, Deng GG, Damaser MS. Combination phosphodiesterase type 4 inhibitor and phosphodiesterase type 5 inhibitor treatment reduces nonvoiding contraction in a rat model of overactive bladder. 2019, 14(8):e0220788.

21. Tsai WH, Wu CH, Yu HJ, Chien CT. I-Theanine inhibits proinflammatory PKC/ERK/ICAM-1/IL-33 signaling, apoptosis, and autophagy formation in substance P-induced hyperactive bladder in rats. Neurourol Urodyn. 2017;36(2):297-307.

22. Wu KC, Chiang BJ, Tsai WH, Chung SD. I-Tiao-Gung extract through its active component daidzin improves cyclophosphamide-induced bladder dysfunction in rat model. 2018, 37(8):2560-2570.

23. Wróbel A, Doboszewska U, Rechberger E, Bańczerowska-Górska M, Czuczwar P, Poleszak E, Dudka J, Wlaź P, Miotła P, Wlaźlak E, et al. Blebbistatin, a Myosin II Inhibitor, Exerts Antidepressant-Like Activity and Suppresses Detrusor Overactivity in an Animal Model of Depression Coexisting with Overactive Bladder. Neurotox Res. 2019;35(1):196-207. 
24. Elrashidy RA, Liu G. Long-term diabetes causes molecular alterations related to fibrosis and apoptosis in rat urinary bladder. Exp Mol Pathol. 2019;111:104304.

25. Chancellor MB. The overactive bladder progression to underactive bladder hypothesis. Int Urol Nephrol. 2014;46(Suppl 1):23-7.

26. Yamaguchi O, Nomiya M, Andersson KE. Functional consequences of chronic bladder ischemia. Neurourol Urodyn. 2014;33(1):54-8.

27. Chien CT, Yu HJ, Lin TB, Chen CF. Neural mechanisms of impaired micturition reflex in rats with acute partial bladder outlet obstruction. Neuroscience. 2000;96(1):221-30.

28. Raivich G, Kreutzberg GW. Nerve growth factor and regeneration of peripheral nervous system. Clinical neurology neurosurgery. 1993;95 Suppl:84-8.

29. Tremp M, Salemi S, Largo R, Andersson KE, Plock JA, Aboushwareb T, Sulser T, Eberli D. Adiposederived stem cells (ADSCs) and muscle precursor cells (MPCs) for the treatment of bladder voiding dysfunction. World J Urol. 2014;32(5):1241-8.

30. Sakuma T, Matsumoto T, Kano K, Fukuda N, Obinata D, Yamaguchi K, Yoshida T, Takahashi S, Mugishima H. Mature, adipocyte derived, dedifferentiated fat cells can differentiate into smooth muscle-like cells and contribute to bladder tissue regeneration. The Journal of urology. 2009;182(1):355-65.

31. Phinney DG, Pittenger MF. Concise Review: MSC-Derived Exosomes for Cell-Free Therapy. Stem Cells. 2017;35(4):851-8.

32. Tyagi P, Smith PP, Kuchel GA, de Groat WC, Birder LA, Chermansky CJ, Adam RM, Tse V, Chancellor MB, Yoshimura N. Pathophysiology and animal modeling of underactive bladder. Int Urol Nephrol. 2014;46(Suppl 1(0 1):11-21.

33. Vinarov A, Atala A, Yoo J, Slusarenco R: Cell therapy for stress urinary incontinence: Present-day frontiers. 2018, 12(2):e1108-e1121.

34. Fraser JK, Hicok KC, Shanahan R, Zhu M, Miller S, Arm DM. The Celution(®) System: Automated Processing of Adipose-Derived Regenerative Cells in a Functionally Closed System. Advances in wound care. 2014;3(1):38-45.

35. Masterson CH, Tabuchi A, Hogan G, Fitzpatrick G, Kerrigan SW, Jerkic M, Kuebler WM, Laffey JG, Curley GF. Intra-vital imaging of mesenchymal stromal cell kinetics in the pulmonary vasculature during infection. Scientific reports. 2021;11(1):5265.

36. Niu LL, Zheng M, Cao F, Xie C, Li HM, Yue W, Gao YH, Bai CX, Zhu SJ, Pei XT. [Migration and differentiation of exogenous rat mesenchymal stem cells engrafted into normal and injured hearts of rats]. Zhonghua yi xue za zhi. 2004;84(1):38-42.

\section{Figures}


a

Common lliac Arteries

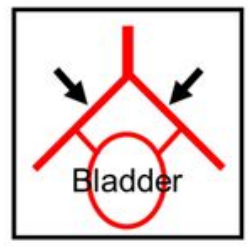

b

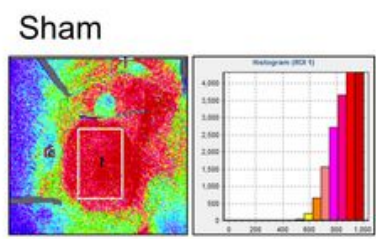

BPAO
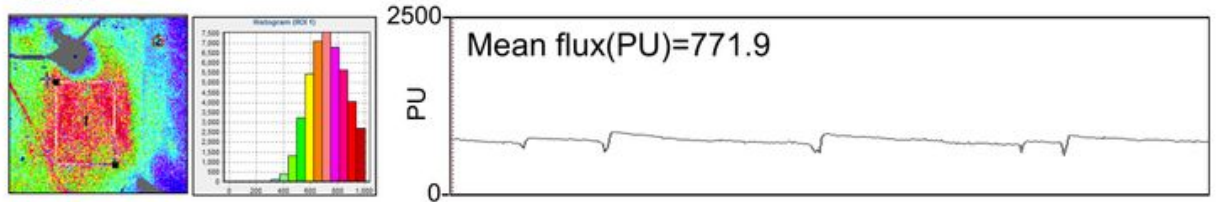

BPAO + ADSCs

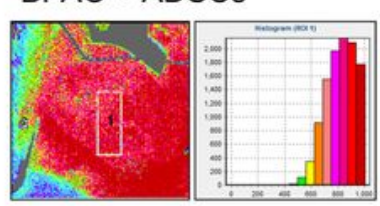

Mean flux(PU)=932.4

ว
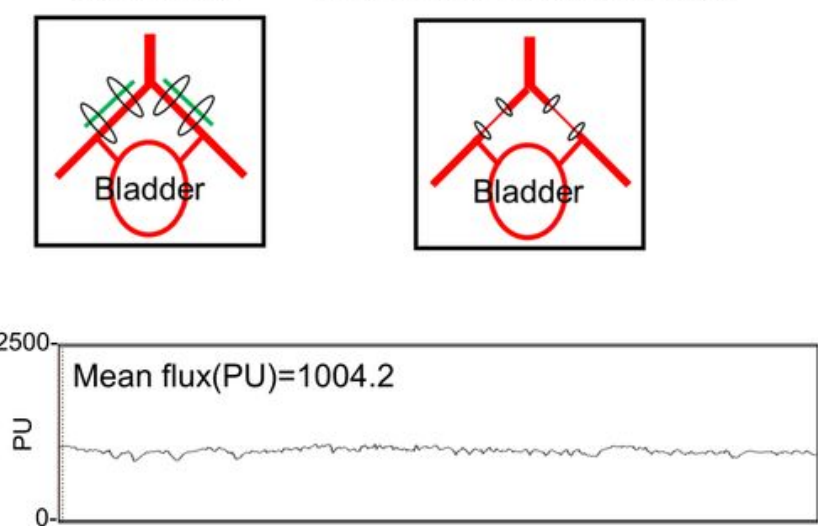

Bilateral Partial Arterial Occlusion

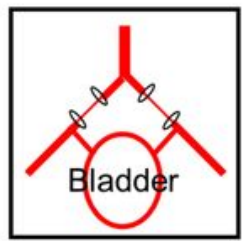

$B P A O+A D S C$-derived MVs

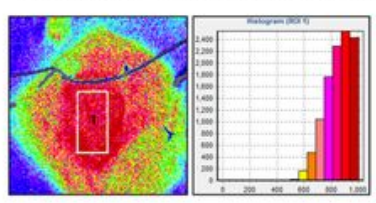

Mean flux(PU)=1022.5

$\supset$

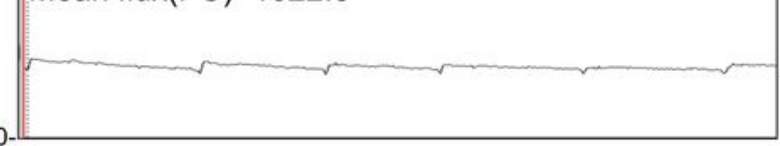

$10 \mathrm{~min}$

C

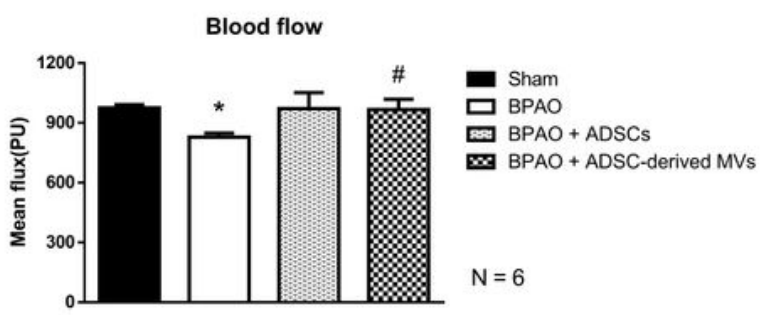

\section{Figure 1}

Demonstration of BPAO and microcirculation patterns on speckle contrast imaging study in separated group. In the top panel, (a) showed the bladder ischemia model, BPAO. In the middle panel, (b) showed the real-time measurements of bladder microcirculation with laser speckle contrast imaging. In the bottom panel, (c) demonstrated a significant redistribution of color-scale of the bladder body associated with a decline in high-flow areas (red) and an increase in low-flow areas in BPAO group compared to 
sham group ( $\left.{ }^{*} p<0.05\right)$. The BPAO+ADSC-derived MVs group had significant mitigation of the reduced microcirculation following BPAO (\# $p<0.05)$.

a

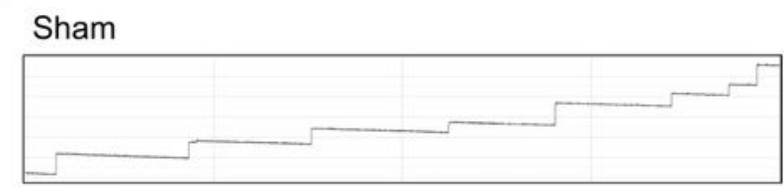

BPAO

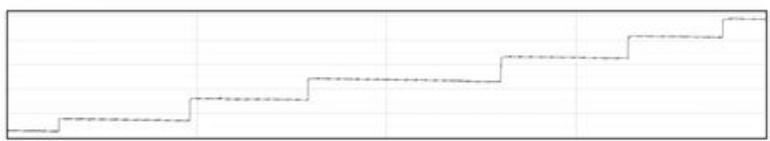

BPAO + ADSCs

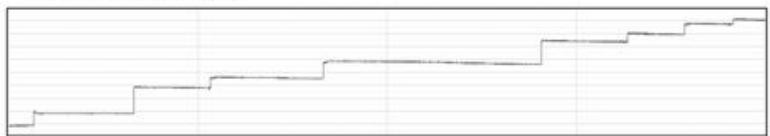

BPAO + ADSC-derived MVs

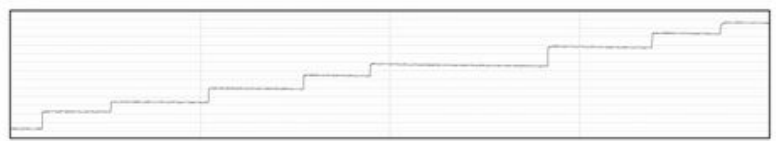

b

3 hours

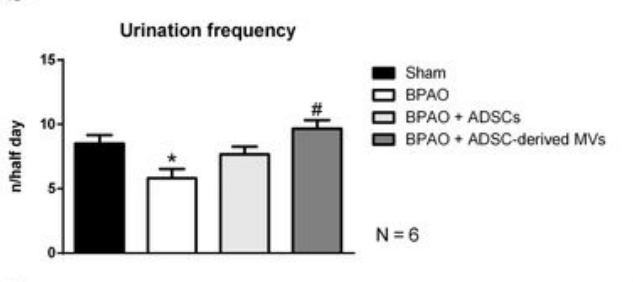

C

Water intake
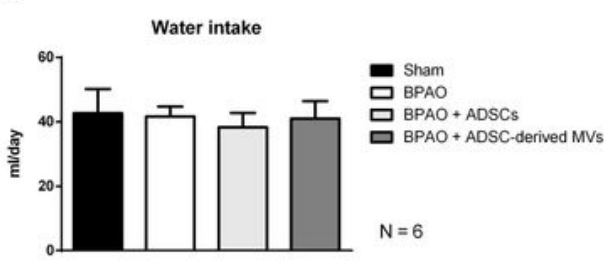

d Food intake

e Urine
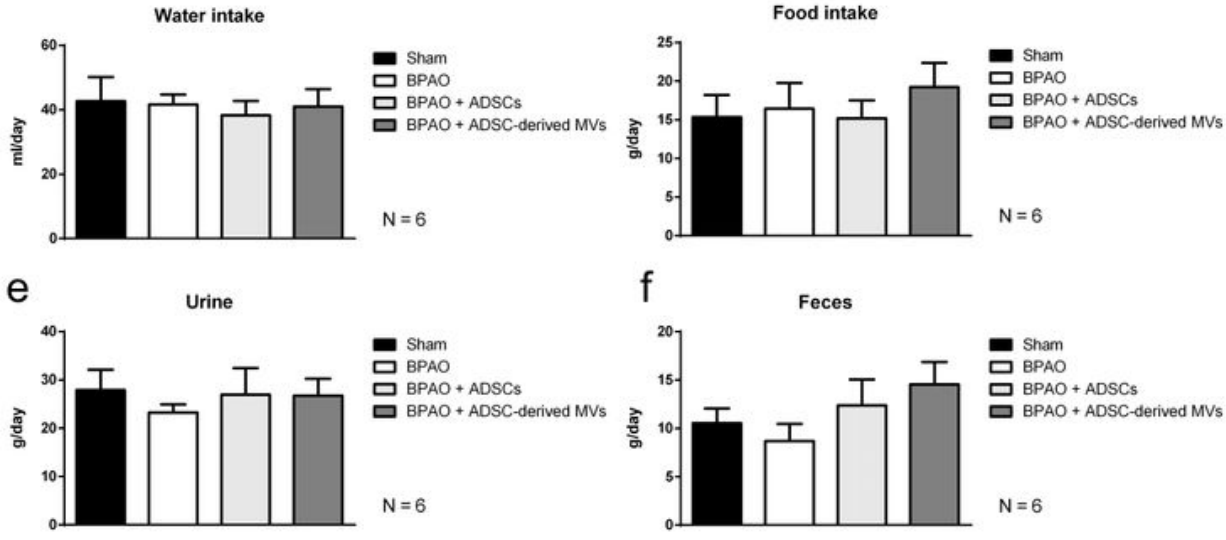

$f$

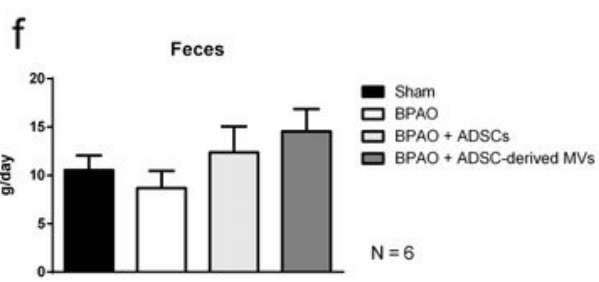

\section{Figure 2}

Physiology parameters on metabolic cage study in separated group. In the upper panel, (a) demonstrated the micturition patterns on metabolic cage study. In the lower panel, $(b-f)$ showed statistical analysis of behavior parameters. (b) showed urinary frequency in BPAO group was significantly lower than sham 
group ( $\left.{ }^{*}<0.05\right)$. Urinary frequency in BPAO+ADSC-derived MVs group were significantly higher than BPAO group (\# $p<0.05)$. The amount of water intake, food intake, urine output, and faces output were of no significant differences among the four groups $(c-f)$.

a
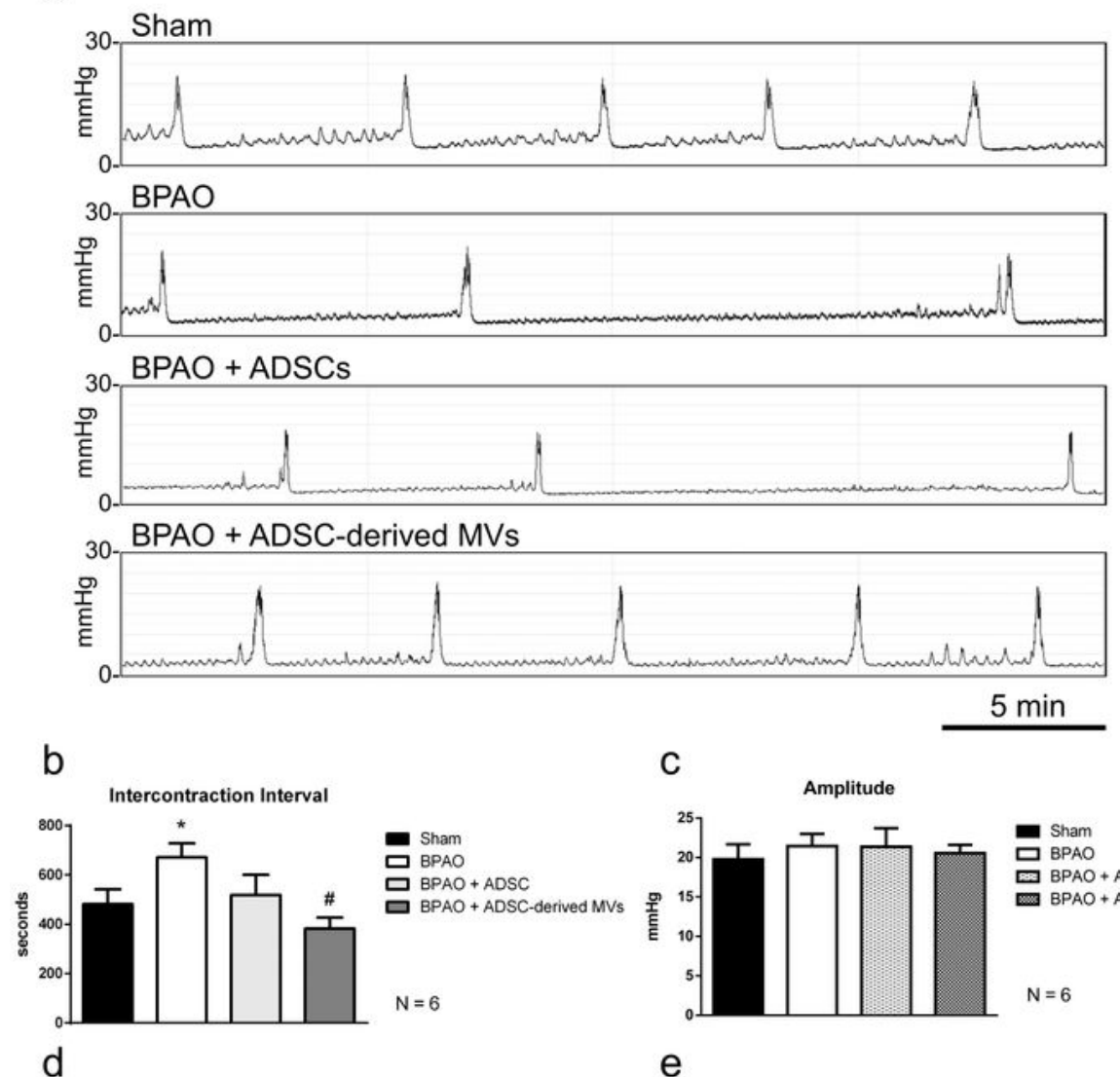

C Amplitude
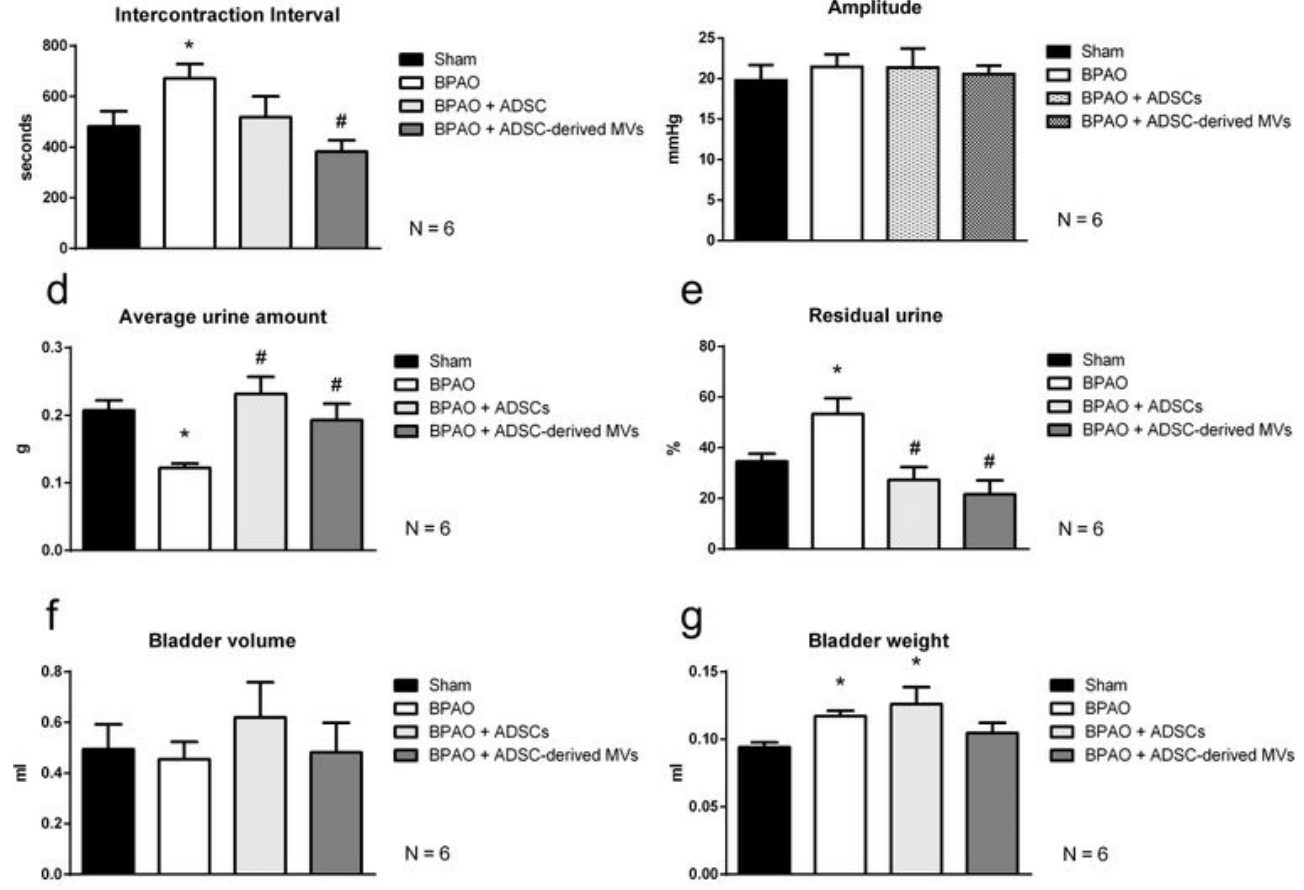

Figure 3

IVP patterns on transcystogram study in separated group. In the top panel, (a) demonstrated the IVP patterns on transcystometrogram. In the lower-left panel, (b) showed significantly higher ICI in BPAO 
group than sham group ( $\mathrm{p}<0.05$ ). The ICI in BPAO+ADSC-derived MVs group is significantly lower than BPAO group (\# $p<0.05)$. In the lower-right panel, (c) showed that the amplitude level is of no significance between groups. (d) showed that the average urine amount in BPAO group significantly lower than sham group (* $p<0.05)$. The average urine amount in BPAO +ADSCs group and BPAO+ADSC-derived MVs group is significantly higher than BPAO group $(\# p<0.05)$. (e) showed that the residual urine amount is significantly higher in BPAO group than sham group ( $\left.{ }^{*} p<0.05\right)$. The residual urine amount in $B P A O+A D S C s$ and BPAO+ADSC-derived MVs group is significantly lower than BPAO group $(\# p<0.05)$. In the bottom-left panel, (f) showed that the bladder volume is of no significant difference, (g) showed that the bladder weight in BPAO group and BPAO+ADSCs group is significantly higher than sham group (* $\mathrm{p}<$ 0.05). 
a
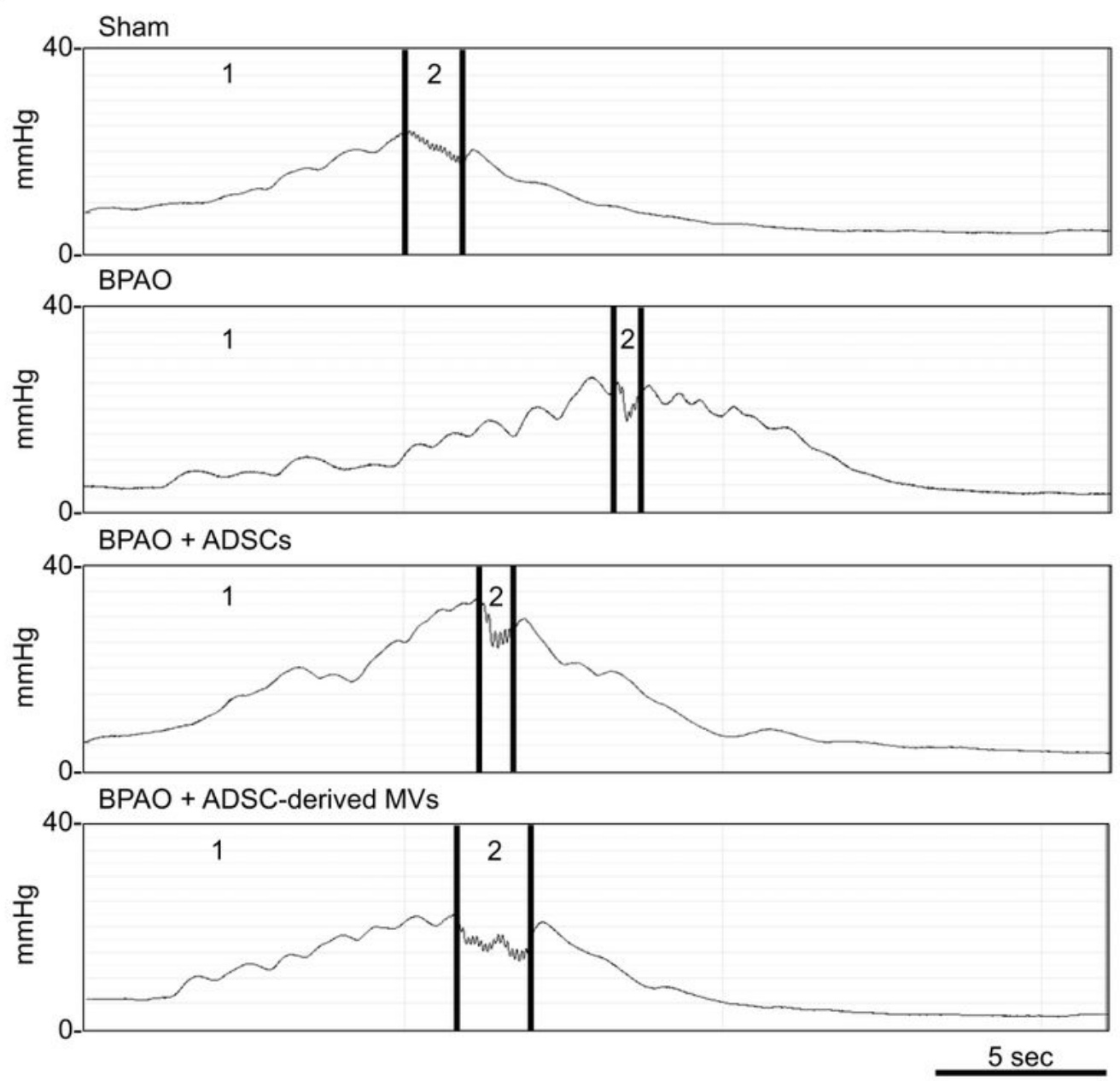

b

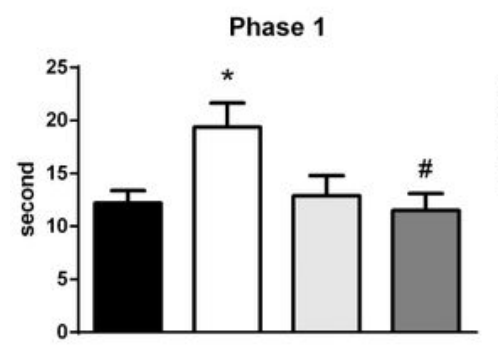

C

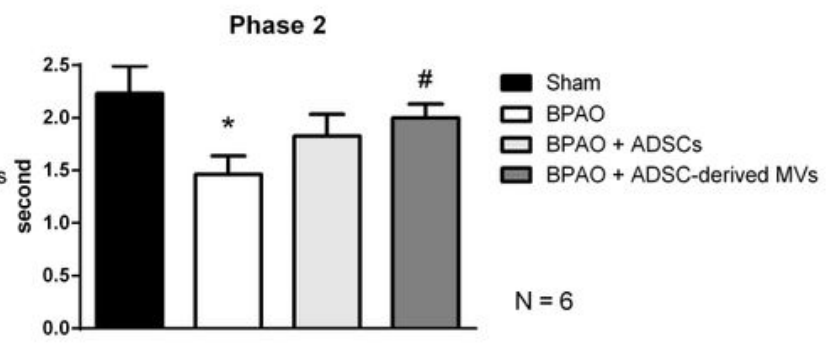

\section{Figure 4}

micturition contraction patterns on transcystogram study in separated group. In the top panel, (a) demonstrated the micturition contraction patterns on transcystometrogram. In the bottom panel, (b) showed the duration of phase 1 contraction in BPAO group is significantly higher than sham group $(* p<$ 0.05). The duration of phase 1 contraction in BPAO+ADSC-derived MVs group in significantly lower than BPAO group $(\# p<0.05)$. (c) showed that the duration of phasic 2 contraction in BPAO group is 
significantly lower than sham group ( $\left.{ }^{*} p<0.05\right)$. The duration of phasic 2 contraction in BPAO+ADSCderived MVs group is significantly higher than BPAO group $(\# p<0.05)$.

a
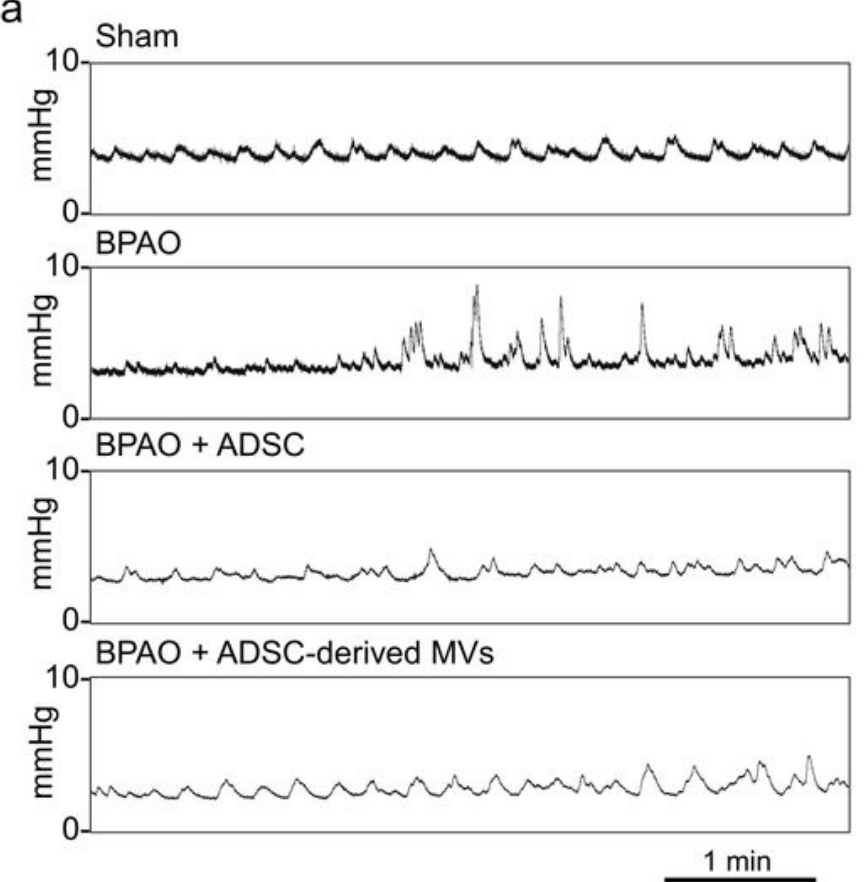

b

C
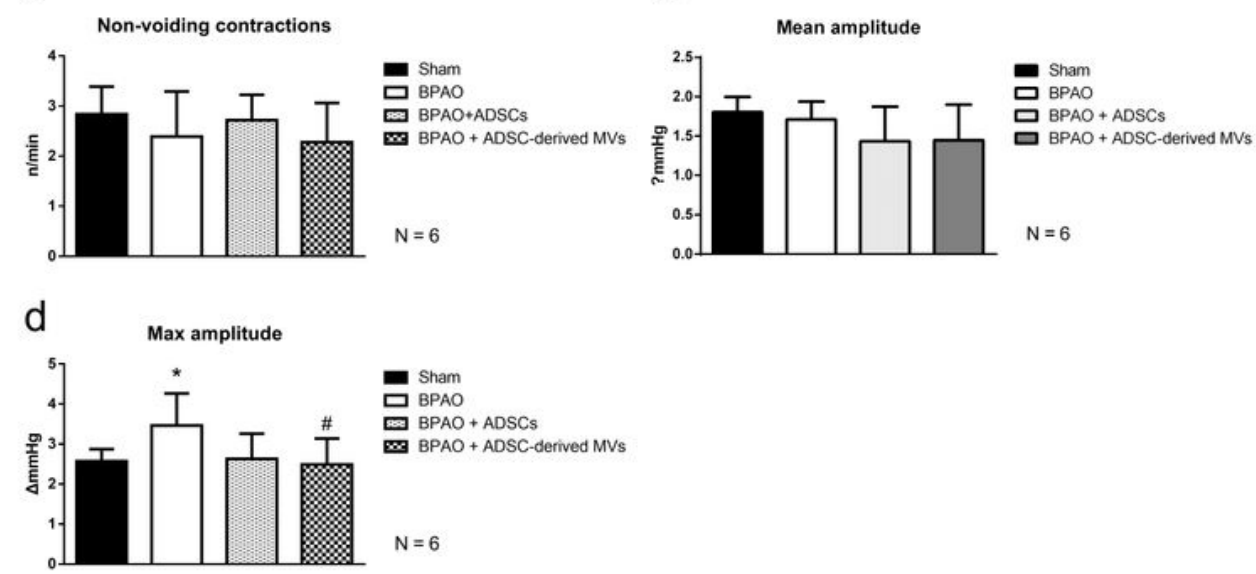

\section{Figure 5}

NVC patterns on transcystogram study in separated group. In the top panel, (a) demonstrated the NVC on transcystometrogram. In the middle-left panel, (b) showed the number of NVC among groups. The number of NVC is of no significant differences between groups. In the middle-right panel, (c) showed the 
mean amplitude is of no significant differences between groups (d) showed the maximum amplitude of NVC. The maximum amplitude of NVC is significantly higher in BPAO group than sham group $\left({ }^{*} p<0.05\right)$. The maximum amplitude of NVC is significantly lower in BPAO+ADSC-derived MVs group than BPAO group $(\# p<0.05)$.
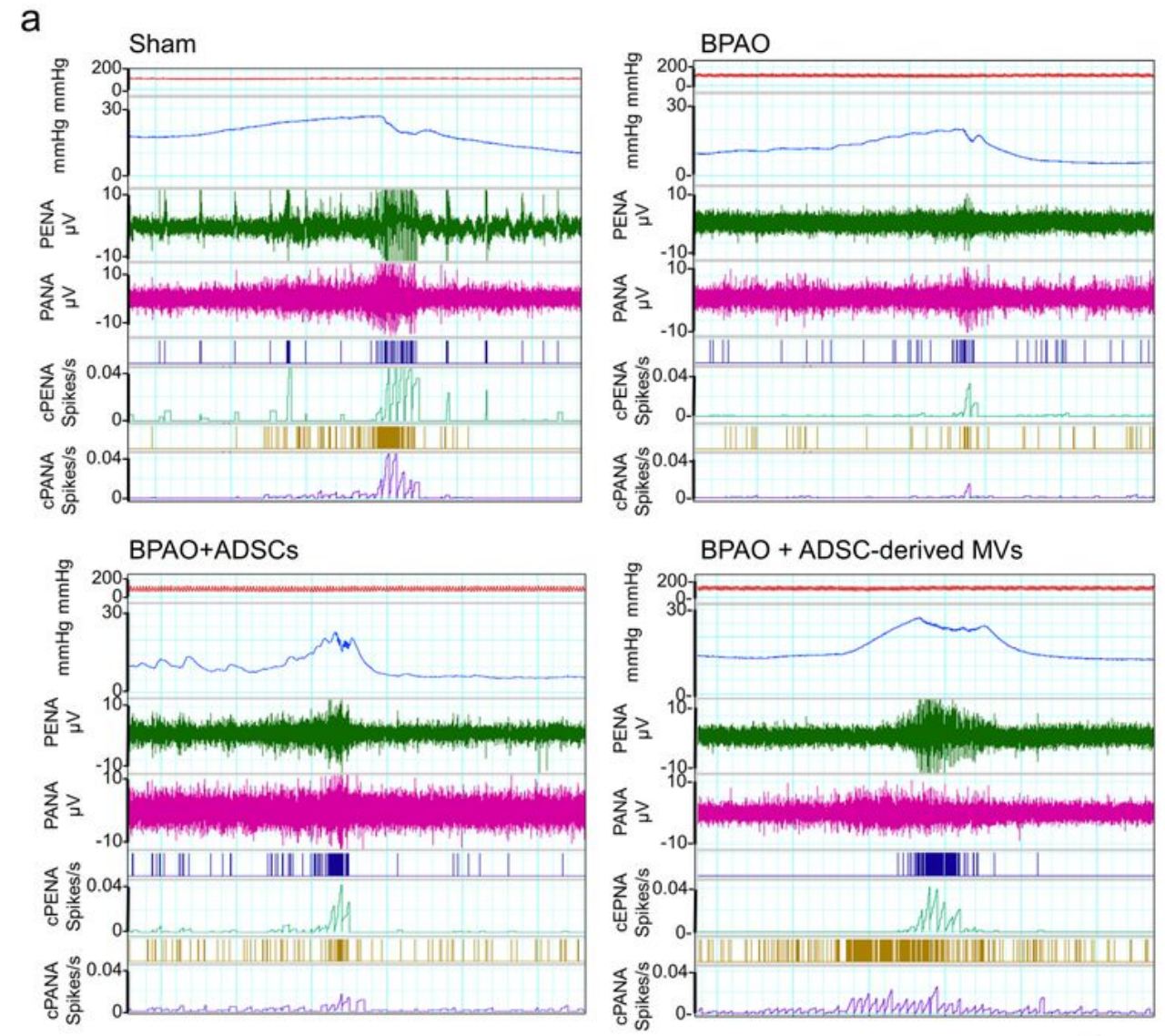

b

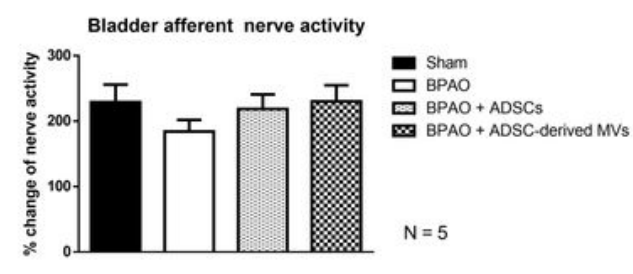

d

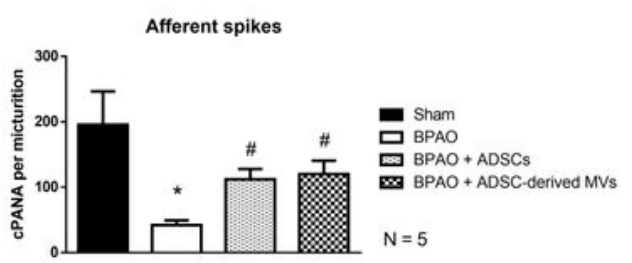

C

e

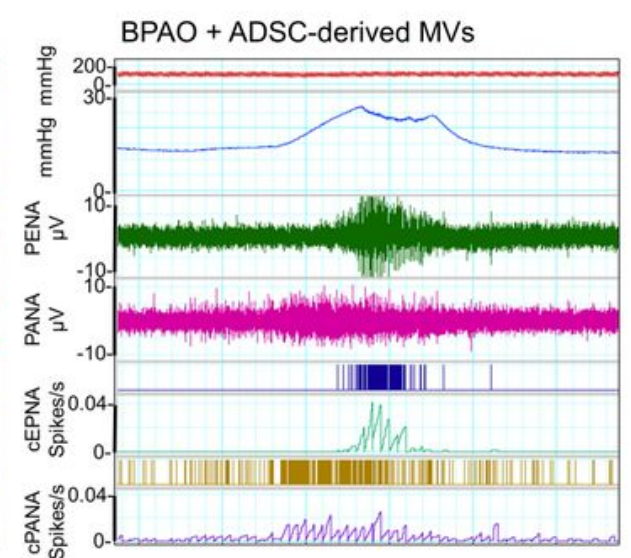

$5 \mathrm{sec}$
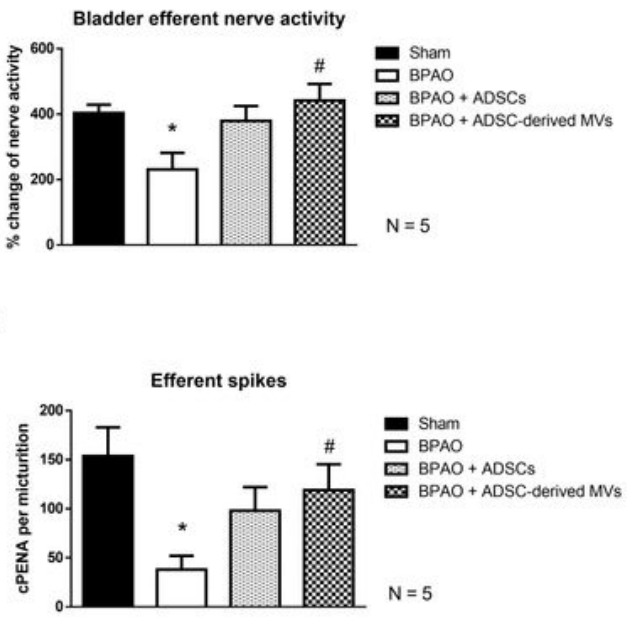

Figure 6 
The arterial blood pressure, pelvic nerve activity on the micturition reflex in separated group. In upper panel, (a) demonstrated the integrated graph recording of ABP $(\mathrm{mmHg}), \operatorname{IVP}(\mathrm{mmHg}), \operatorname{PENA}(\mu \mathrm{v})$, PANA( $\mu \mathrm{v}), \mathrm{cPENA}$ (spikes/s), cPANA (spikes/s). (b) showed the changes of of PANA ( $\mu \mathrm{v})$ micturition phase and baseline had a trend of lower in BPAO group $(p>0.05)$. (c) showed the changes of PENA $(\mu v)$ micturition phase and baseline was significantly lower in BPAO group $\left({ }^{\star} \mathrm{p}<0.05\right)$. The changes of PENA $(\mu \mathrm{v})$ micturition phase and baseline is significantly higher in BPAO+ADSC-derived MVs group $(\# p<0.05)$ (d) The CPANA per micturition in BPAO group was significantly lower than that in sham group $\left({ }^{*} p<0.05\right)$. The CPANA per micturition in BPAO+ADSCs group and BPAO+ADSC-derived MVs was significantly higher than sham group $(\# p<0.05)$. (e) The cPENA per micturition in BPAO group was significantly lower than that in sham group ( $\left.{ }^{*} \mathrm{p}<0.05\right)$. The cPENA per micturition in BPAO+ADSC-derived MVs was significantly higher than sham group $(\# p<0.05)$. 

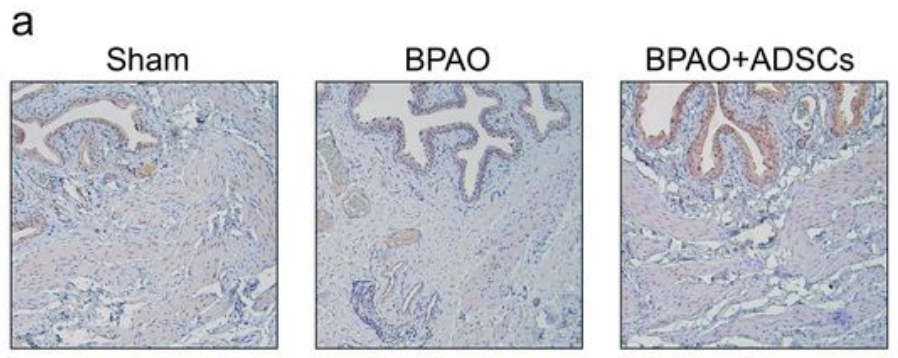

BPAO + ADSC-derived MVs
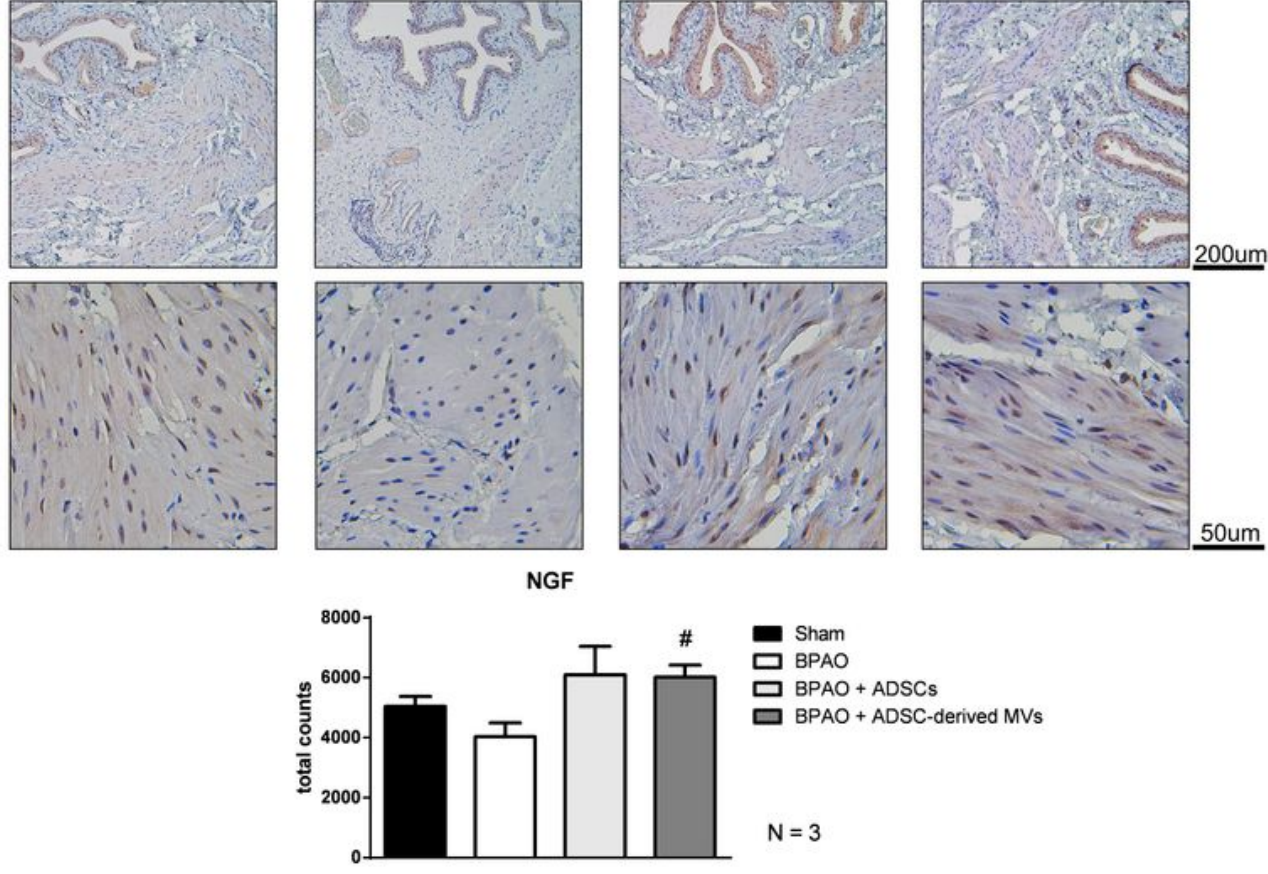

b

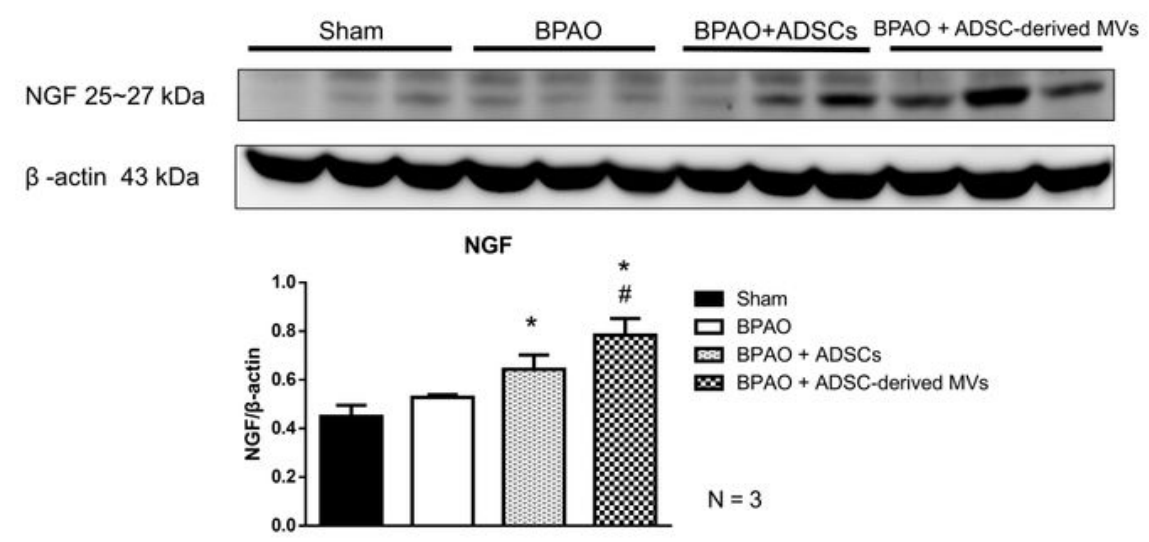

\section{Figure 7}

NGF staining and expression in separated group. In the top panel, (a) showed the NGF staining and image analysis of NGF staining. It showed the NGF density in BPAO+ADSC-derived MVs group was significantly higher than BPAO group (\# $\mathrm{p}<0.05)$. The NGF density in BPAO+ADSCs group had a trend of higher than BPAO group ( $p$ > 0.05). In the bottom panel, $(b)$ showed the western blot of NGF level. It showed significantly higher level of NGF expression in BPAO+ADSCs group and BPAO+ADSC-derived 
MVs group compared to sham groups ( $\left.{ }^{*}<<0.05\right)$. The level of NGF expression is higher in BPAO+ADSCderived MVs group than BPAO group (\# $\mathrm{p}<0.05)$.

a

$\mathrm{P} 2 \mathrm{X}_{2}$ receptor, $48 \mathrm{kDa}$

$\beta$-actin, $43 \mathrm{kDa}$

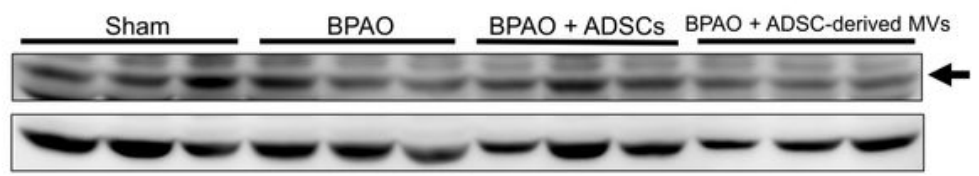

P2X Receptor

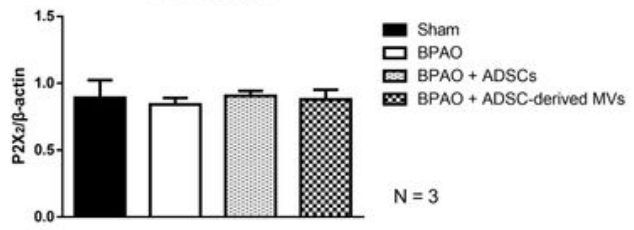

b
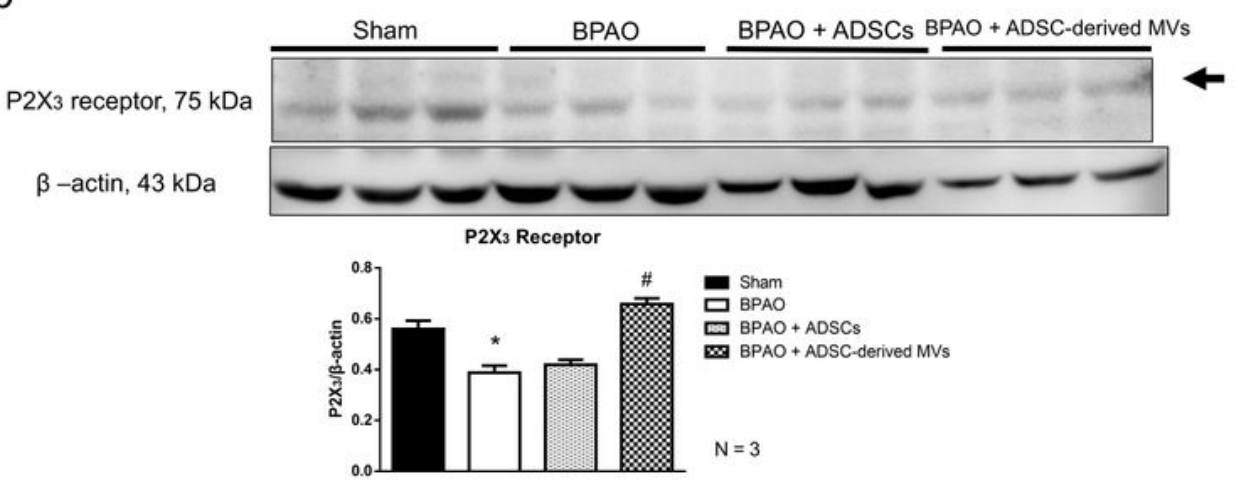

C

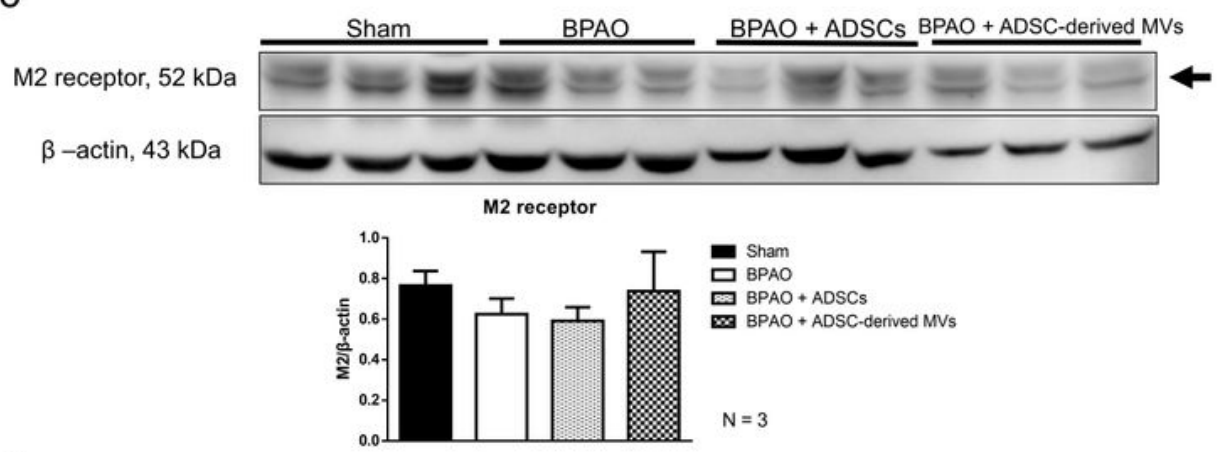

d

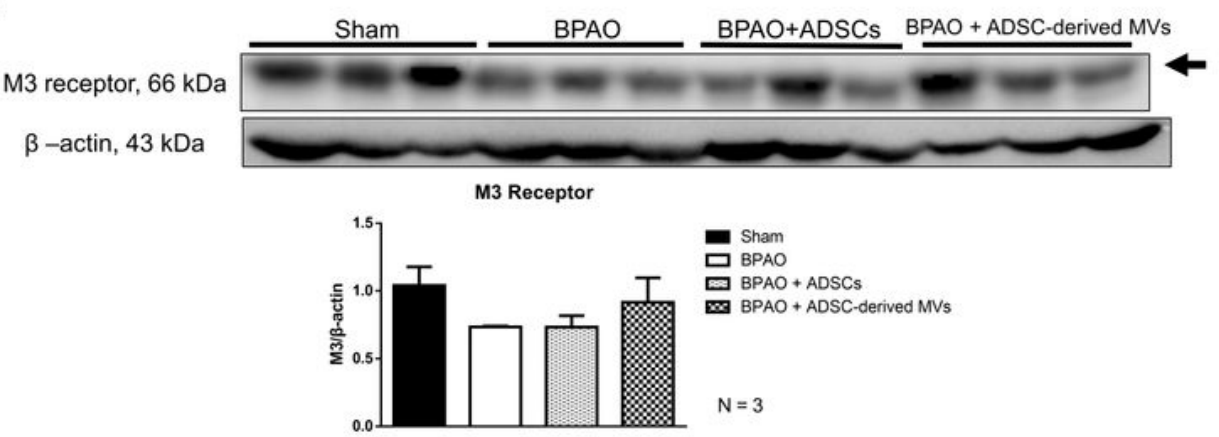

Figure 8

showed the expression of purinergic receptor proteins (P2X2 and P2X3) and muscarinic cholinergic (M2 and M3) by western blotting. In the top panel, (a) shows that the expression of P2X2 receptor is not significantly different, and (b) shows that the expression of P2X3 receptor was significantly decreased in 
the BPAO group compared to the sham group $(* p<0.05)$. The expression of P2X3 receptor was significantly increased is significantly increased in the BPAO+ADSC-derived MVs group compared to the BAPO group (\# $p<0.05$ ). (c) shows that the expression of $M 2$ receptors was not significantly different, and (d) shows that the expression of M3 receptors was not significantly different.

a
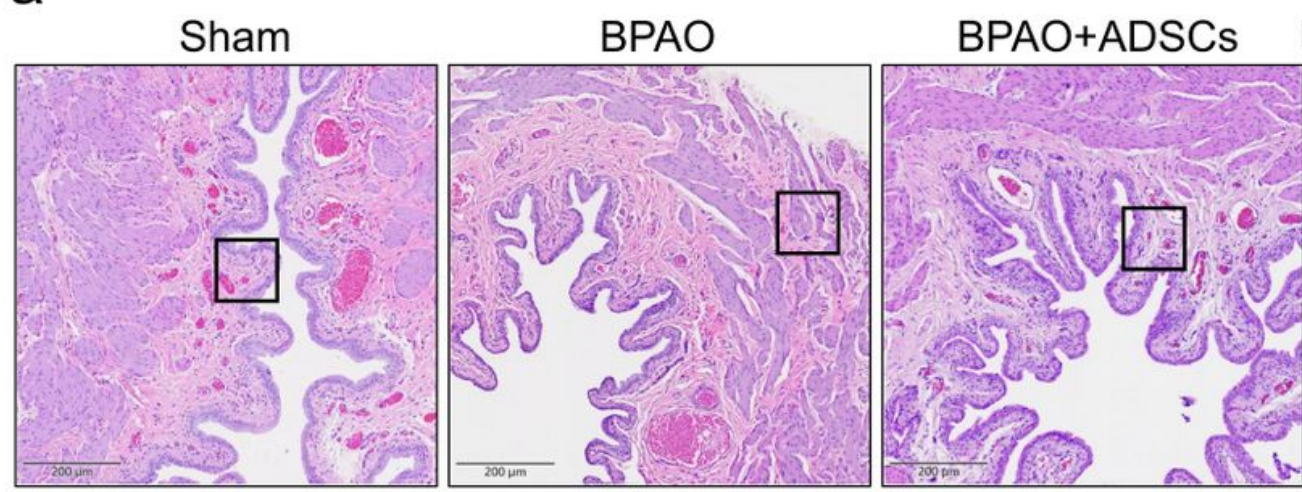

BPAO + ADSC-derived MVs
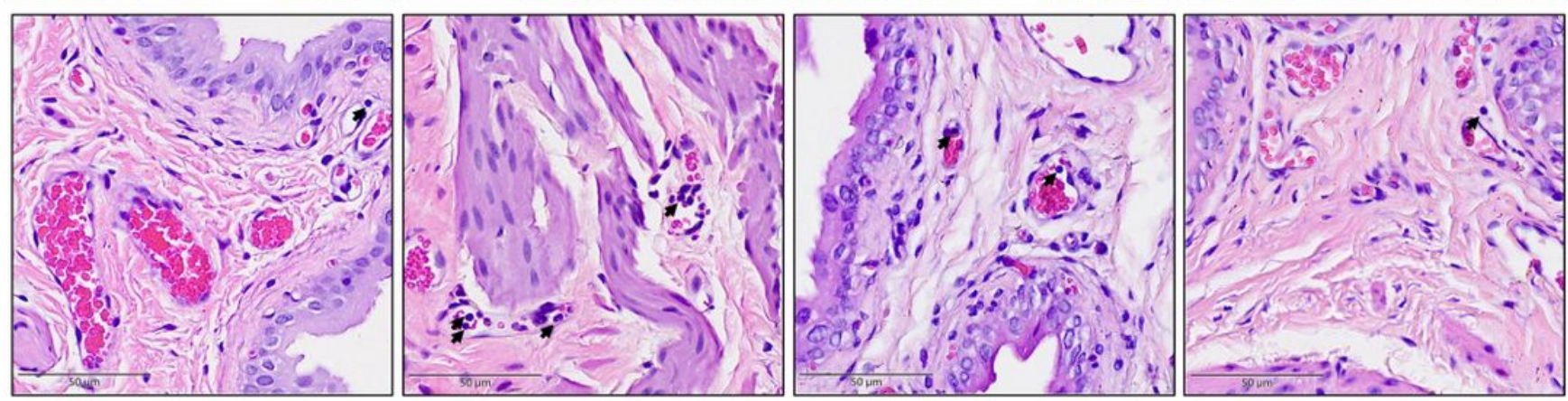

200 um

b

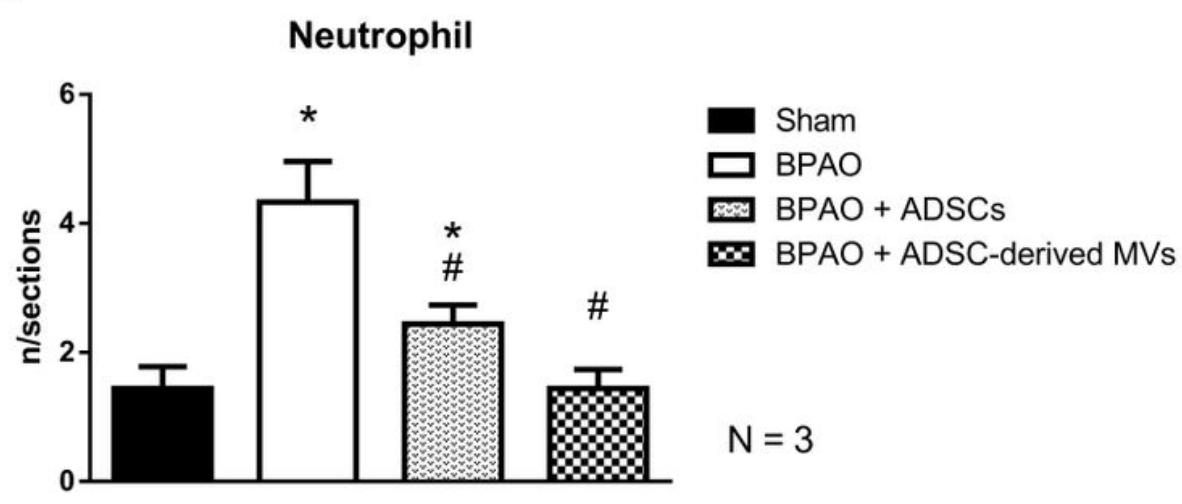

Figure 9

Histological staining and analyses in separated group. In the top panel, (a) showed the H\&E staining among groups. In the bottom panel, (b) showed analysis of neutrophil counts. The results showed the neutrophil counts in BPAO group and BPAO+ADSCs group are significantly higher than sham group $\left({ }^{*} \mathrm{p}<\right.$ 
0.05). The neutrophil counts of BPAO+ADSC-derived MVs group are significantly lower than BPAO group $(\# \mathrm{p}<0.05)$.

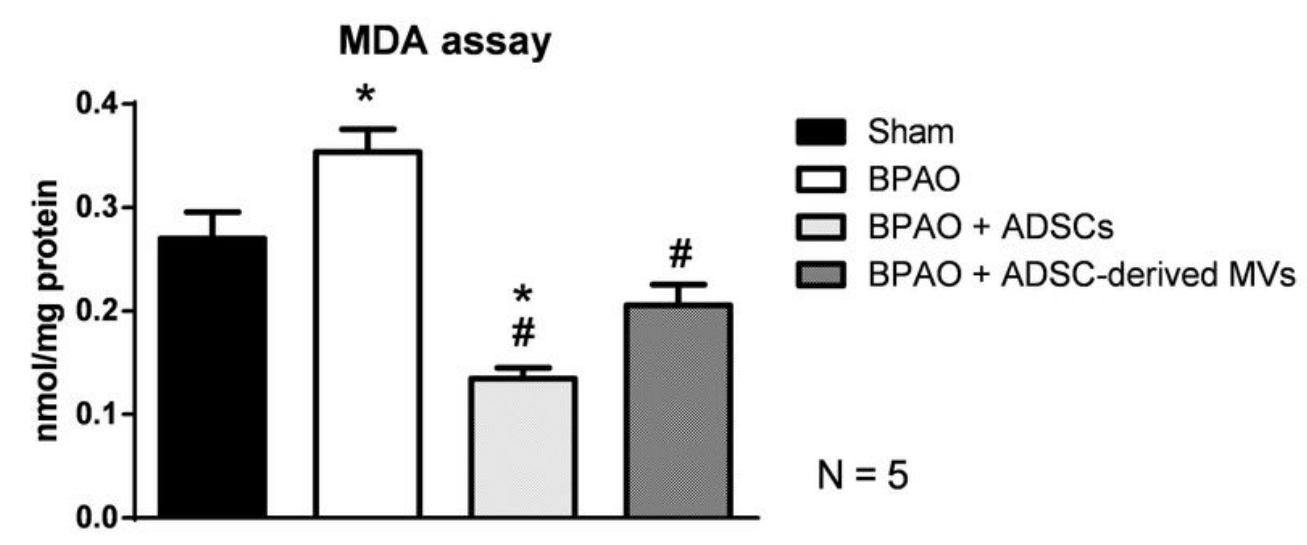

Figure 10

MDA assay in separated group. It showed BPAO group had significantly higher level of MDA than sham group ( $\left.{ }^{*}<0.05\right)$. The MDA level is significant lower in BPAO+ADSCs group than sham group $\left({ }^{*} p<\right.$ 0.05). The MDA level is significant lower in BPAO+ADSCs group and BPAO+ADSC-derived MVs group than BPAO group $(\# p<0.05)$. 
a

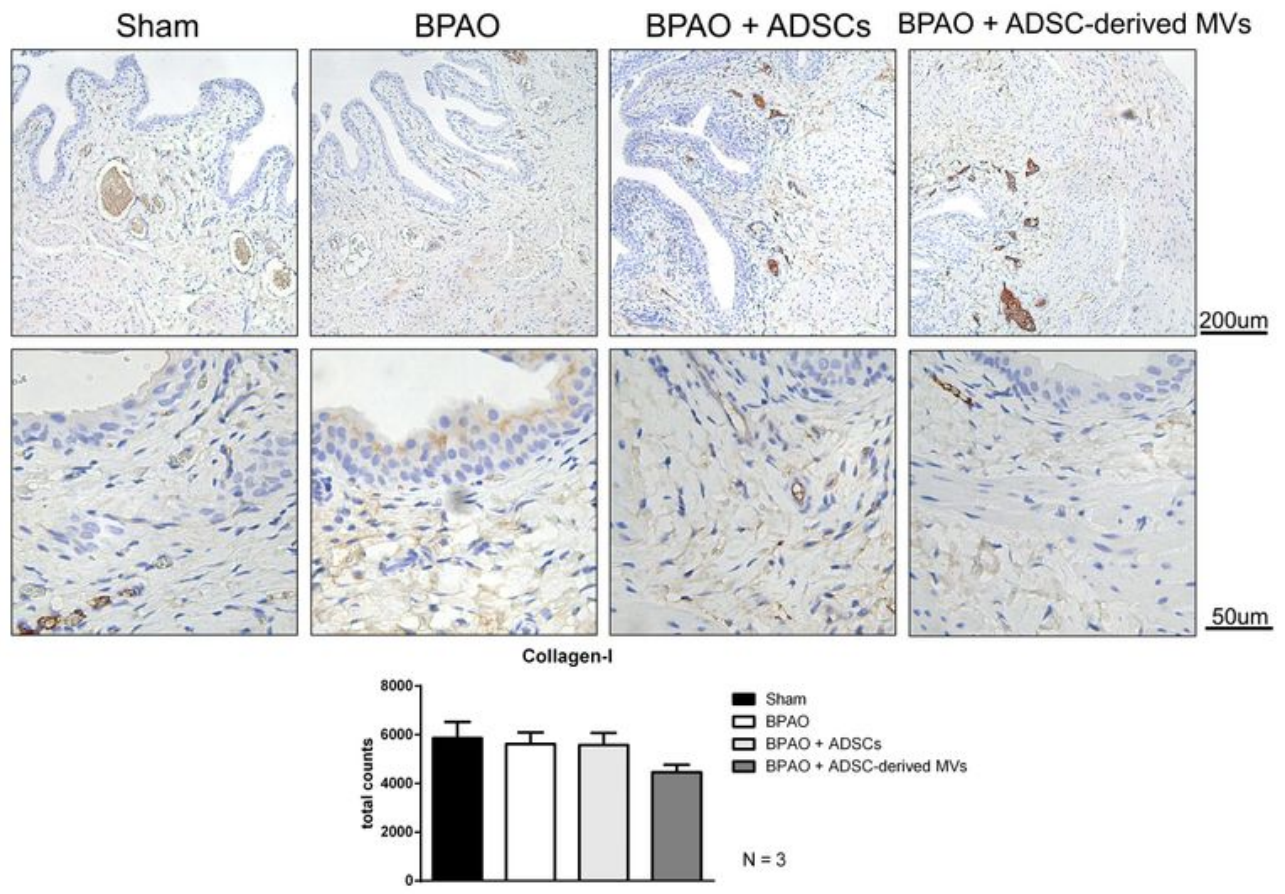

b

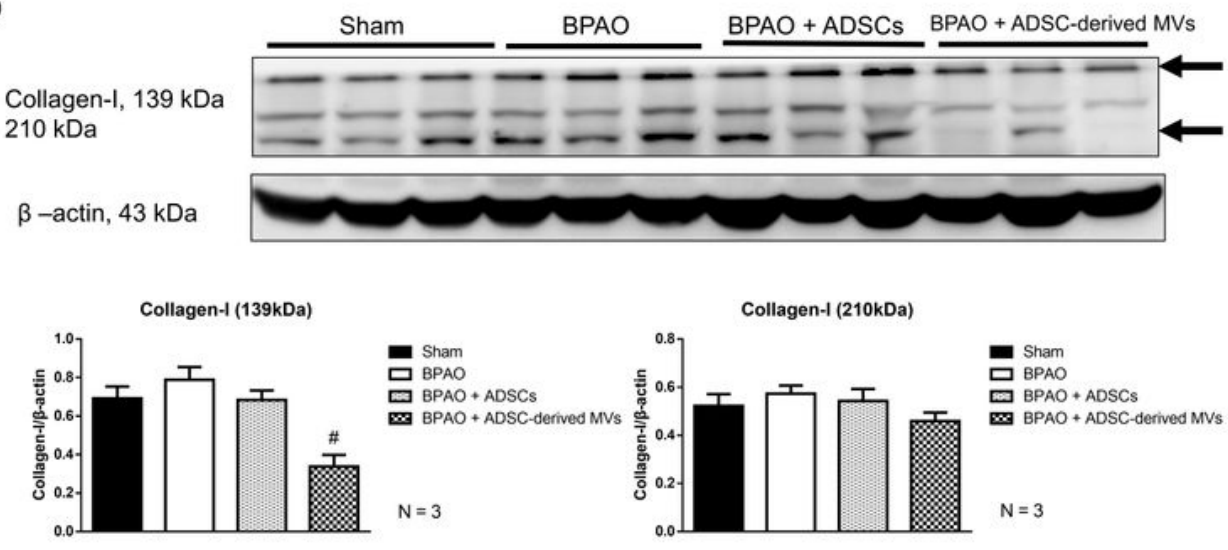

\section{Figure 11}

Collagen-1 staining and expression in separated group. In the top panel, (a) showed the Collagen-1 staining and image analysis of Collagen-1 staining. It showed the Collagen-1 density in BPAO+ADSCderived MVs group had a trend of lower than the other groups ( $p>0.05$ ). In the bottom panel, (b) showed the western blot of Collagen-1 level. It showed the level of Collagen-1 (139 kDa) expression in and BPAO+ADSC-derived MVs group was significantly lower than BPAO group (\# $p<0.05)$. The level of 
Collagen-1 (210 kDa) expression in and BPAO+ADSC-derived MVs group had a trend of lower lower than BPAO group $(p>0.05)$. 